\title{
Impact of Heat and Mass Dispersion and Thermal Effects on the Scale-up of Monolith Reactors
}

\author{
Tian Gu and Vemuri Balakotaiah* \\ Department of Chemical and Biomolecular Engineering, University of Houston, Houston, TX 77204, USA
}

\begin{abstract}
Monolith reactors used in catalytic after-treatment systems (e.g. TWC, DOC, LNT and SCR) are often studied in laboratory experiments using monolith samples of the same cell density, washcoat thickness, and catalyst loading but with a smaller length compared to the fullscale reactor. The flow rate in the laboratory scale operation is selected so that the same space velocity is maintained as in the full-scale system. However, matching space velocity between the laboratory-scale and full-scale reactor leads to similar performance only under ideal conditions such as isothermal or adiabatic operation with negligible heat and mass dispersion (i.e. plug flow conditions) and small adiabatic temperature rise. In this work, it is shown that when thermal effects are significant (or adiabatic temperature rise is large) and heat and mass transfer and dispersion effects are not negligible, similarity may not exist and the ignition/extinction behavior of the two systems can be qualitatively different. Further, when laboratory-scale data is used for kinetic parameter estimation, failure to consider these effects in the reactor model can lead to false kinetic parameters and inaccurate prediction of reactor performances. Another major reason for possible discrepancy between laboratory-scale and full-scale reactors, external heat exchange with furnace (surroundings), is also investigated. We present analysis, simulations, and analytical results quantifying the impact of these effects on the scale-up of monolith reactors.

Keywords: Catalytic after-treatment; Monolith reactor; Scale-up; Bifurcation diagram; Hysteresis; Light-off
\end{abstract}

\footnotetext{
*Corresponding author. E-mail address: bala@uh.edu (V. Balakotaiah)
} 


\section{Introduction and literature review}

The monolith reactor is the most widely used man-made reactor in the world. While the exact number of units is unknown, it is estimated that there are about one billion monolith reactors used in the treatment of emissions from automobiles alone (e.g. three-way converters, TWCs; diesel oxidation converters, DOCs; lean-NOx traps, LNTs; selective catalytic reduction units, SCRs; ammonia slip converters, ASCs and so forth.) It consists of a honeycomb structure with a large number of straight parallel channels of small hydraulic diameter $(0.5-6 \mathrm{~mm})$ through which the fluid flows. The substrate of the monoliths used in automobile exhaust after-treatment is made up of either ceramic, which has lower thermal conductivity $\left(0.8-2 \mathrm{~W} \cdot \mathrm{m}^{-1} \cdot \mathrm{K}^{-1}\right)$, or metallic, which is made up of ferritic steels with higher thermal conductivity $\left(10-30 \mathrm{~W} \cdot \mathrm{m}^{-1} \cdot \mathrm{K}^{-1}\right.$.) The catalytic materials (alumina washcoat containing precious metals such as $\mathrm{Pt} / \mathrm{Pd} / \mathrm{Rh}$ and storage materials such as $\mathrm{Ce} / \mathrm{Ba}$ or zeolite particles mixed with binders) are deposited on the substrate in the form of one or more layers with thickness in the range of $10-50 \mu \mathrm{m}$ for each layer. In addition to their use in pollution abatement, monolith reactors are also finding applications in the chemical industry as a replacement for the traditional packed-bed reactor.

The performance of various catalyst formulations in treating the emissions from automobiles is often tested in laboratory experiments using monolith samples of the same cell density, washcoat layer thickness, and catalyst composition and loading but with a smaller length scale (typically $1-2 \mathrm{~cm}$ in length) compared to full-scale systems $(10-30 \mathrm{~cm})$. The flow rate in the laboratory-scale operation is selected such that the space velocity is the same as that in the full-scale system [In addition to various measurement conveniences, the smaller length and flow rate used in laboratory-scale operation is to minimize the amount of synthetic exhaust gases used and hence the cost of experiments.] It is hoped that matching the catalyst geometrical properties and space velocity ensures similarity between the laboratory-scale and full-scale systems. However, the conditions for similarity between these two systems have not been validated with any analysis or computation or direct experimental comparison. This lack of validation and the interest of the CLEERS [1] after-treatment community in the scale-up of these reactors, is the motivation for this study.

While the general principles of scale-up in engineering are well known [2] (e.g. matching of various 
dimensionless groups impacting the process), application requires the identification of all the relevant independent variables, which are case dependent. In the context of catalytic reactors in which the same catalyst and fluid is used, it is known that the performance of reactors with different physical scales could be different for the following reasons: (i) fluid flow patterns are not similar (ii) heat and mass dispersion effects are different (iii) the concentrations and temperature ranges used are different, and (iv) the external conditions such as heat exchange with surroundings are different at the two scales.

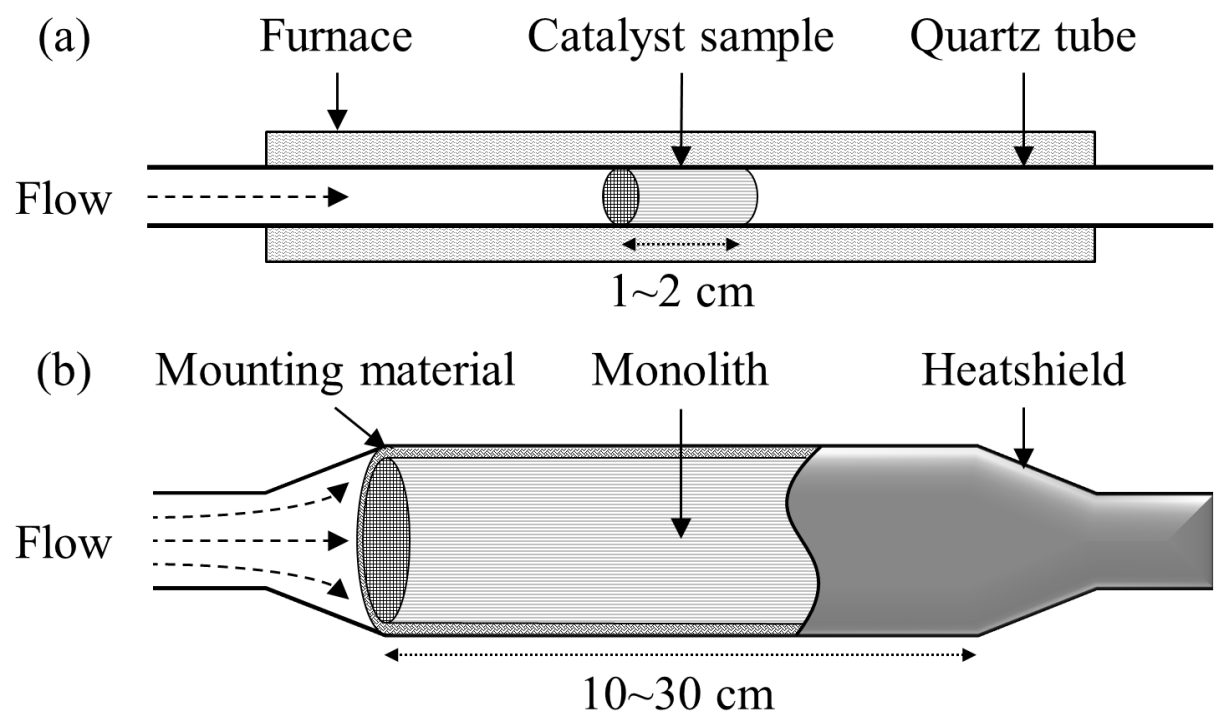

Figure 1: Schematic diagrams of (a) a lab-scale reactor and (b) a full-scale reactor.

The main goal of this work is to analyze in some detail the impact of heat and mass dispersion, thermal effects (adiabatic temperature rise or use of different inlet concentrations) and heat losses from the reactor on the scale-up of monolith reactors used in the exhaust after-treatment as well as other applications. This paper is organized as follows. In the next section, we review briefly the detailed as well as simplified or coarse-grained models of monolith reactors and the relationship between various dimensionless groups that appear in these models. In Section 3, we examine the impact of mass dispersion on the scale-up of isothermal reactors with linear as well as nonlinear kinetics. In Section 4, we examine the impact of heat dispersion and adiabatic temperature rise (or use of different inlet concentration) on the ignition and extinction behavior and compare the light-off curves of laboratory-scale reactors with those of full-scale reactors. In Section 5, we use a one-dimensional two-phase version of the model to examine the influence of interphase gradients. In 
Section 6, we examine the impact of heat losses or exchange from the reactor to surroundings on the scale-up. In the last section, we summarize the main contributions of this work and present some recommendations.

\section{Models of monolith reactors}

As we have assumed hydrodynamic similarity between the two scales, we need to consider only species and energy balance equations in order to examine the impact of heat and mass dispersion and thermal effects on the scale-up behavior. For the adiabatic case, this can be done by considering the model of a single channel provided the flow distribution to the various channels is identical. Fig. 2a shows a schematic diagram of the cross-section of a monolith channel with a single washcoat layer. Other than square-shaped channels, which are most commonly found, ceramic monolith reactors are also made with triangular and hexagonal channels for either higher mechanical strength or lower back pressure [4]. Bhattacharya et al. [5] showed that channels of various shapes can be treated by a single model through proper shape normalization. Using effective washcoat thickness and assuming azimuthal symmetry, monolith channel of arbitrary shape may be treated as a circular channel, and a two-dimensional model is obtained. In this work, we assume that geometric similarity exists and consider an equivalent axisymmetric system with a circular flow channel and uniformly thick washcoat and wall layers (as shown in Fig. 2b). This approximation is justified since we have already assumed that geometric similarity exists between the channels of the two scales and the differences are mainly due to axial length scales. Thus, we start from a detailed two-dimensional (axisymmetric) model to determine and compare the relevant dimensionless groups in both lab-scale and full-scale systems. Based on this comparison, the model is further simplified by lumping the various dimensionless groups that are not matched in lab- and full-scale systems. In this way, we can examine all the quantitative and qualitative differences between two scales without introducing unnecessary complexity in the mathematical model. 
(a)
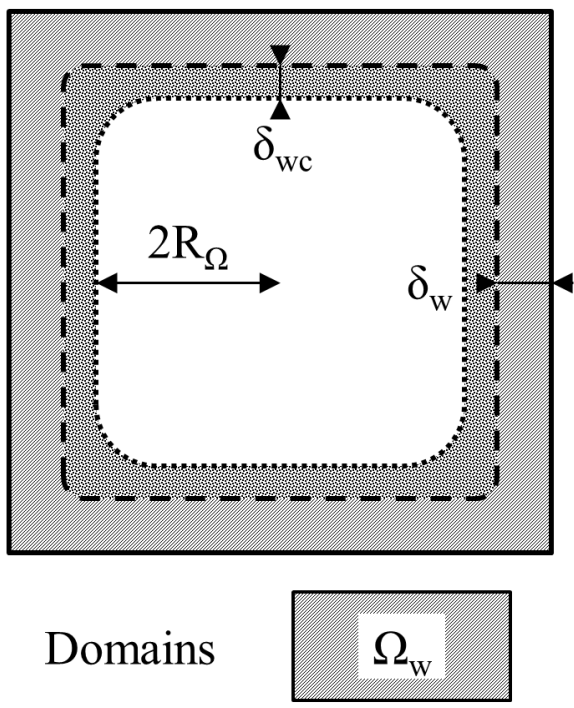

Boundaries (b)
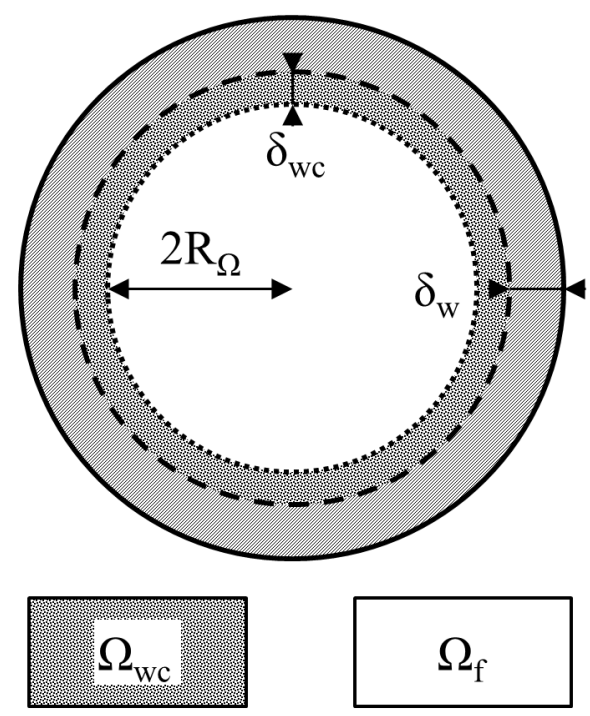

$---\partial \Omega_{\mathrm{wc}}$

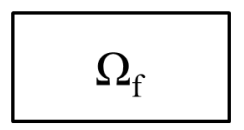

$\partial \Omega_{\mathrm{f}}$

Figure 2: Schematic diagram of the cross-section of (a) a single monolith channel and (b) axisymmetric approximation for modeling.

\subsection{Two-dimensional monolith model}

As shown in Fig. 2, the monolith channel is divided into three domains - flow channel $\left(\Omega_{f}\right)$, catalytic washcoat $\left(\Omega_{w c}\right)$ and substrate wall $\left(\Omega_{w}\right)$. The feed gas flows axially through the monolith channel while mass and heat are transported in both axial and radial directions by convection and diffusion. We distinguish between the washcoat and the substrate wall because heat is only generated in the washcoat where reactions take place. The model can be applied to the case of multiple species and reactions, but here we consider only one limiting reactant with one reaction because the focus is to compare reactor performances instead of kinetic models.

The model equations are derived from species and energy balances in each domain. The species balance in the flow channel is given by

$$
\frac{\partial C_{f}}{\partial t}+u(r) \frac{\partial C_{f}}{\partial x}=D_{f} \nabla_{\perp}^{2} C_{f}+D_{f} \frac{\partial^{2} C_{f}}{\partial x^{2}} \text { in } \Omega_{f}, 0<x<L, t>0
$$


The species balance in the washcoat is

$$
\epsilon_{w c} \frac{\partial C_{w c}}{\partial t}=D_{w c} \nabla_{\perp}^{2} C_{w c}+D_{w c} \frac{\partial^{2} C_{w c}}{\partial x^{2}}-R\left(C_{w c}, T_{w c}\right) \text { in } \Omega_{w c}
$$

Here, $C_{f}$ and $C_{w c}$, represent reactant concentrations in the fluid phase and the washcoat, respectively. The species balance in the substrate wall is not considered since there is no reaction in that domain and the molecular diffusivity in the substrate is much smaller than other domains. Here, $R\left(C_{w c}, T_{w c}\right)$ is the reaction rate per unit volume of the washcoat; $D_{f}, D_{w c}$ are molecular diffusivities of the reactant in the fluid phase and the washcoat, respectively; $L$ is the length of the reactor and $\epsilon_{w c}$ is the porosity of the washcoat. Assuming the flow is laminar and fully developed, the fluid velocity profile $u$ is only a function of the radial position $r$,

$$
u(r)=2 \bar{u}\left(1-\frac{r^{2}}{4 R_{\Omega}^{2}}\right), 0<r<2 R_{\Omega}
$$

in which $\bar{u}$ is the average fluid velocity and $R_{\Omega}$ is the hydraulic radius of the flow channel $\Omega_{f}$ (half of the geometric radius for a circular channel). The initial and boundary conditions for the species balances are given by

$$
\begin{gathered}
C_{f}=C_{f}^{0}(x, r), C_{w c}=C_{w c}^{0}(x, r) \text { at } t=0 . \\
D_{f} \mathbf{n}_{\Omega_{f}} \cdot \nabla_{\perp} C_{f}=D_{w c} \mathbf{n}_{\Omega_{f}} \cdot \nabla_{\perp} C_{w c}, C_{f}=C_{w c} \text { on } \partial \Omega_{f}, \\
\mathbf{n}_{\Omega_{w c}} \cdot \nabla_{\perp} C_{w c}=0 \text { on } \partial \Omega_{w c}, \\
D_{f} \frac{\partial C_{f}}{\partial x}=u\left(C_{f}-C_{f}^{i n}(t)\right), \frac{\partial C_{w c}}{\partial x}=0 \text { at } x=0, \\
\frac{\partial C_{f}}{\partial x}=\frac{\partial C_{w c}}{\partial x}=0 \text { at } x=L .
\end{gathered}
$$

Here, $\nabla_{\perp}$ and $\nabla_{\perp}^{2}$ are transverse gradient and Laplacian operators; $\mathbf{n}_{\Omega_{f}}$ and $\mathbf{n}_{\Omega_{w c}}$ are unit normal vectors radially outward to the fluid-washcoat interface $\partial \Omega_{f}\left(r=2 R_{\Omega}\right)$ and the washcoat-substrate interface $\partial \Omega_{w c}\left(r=2 R_{\Omega}+\delta_{w c}\right)$, respectively. Bold symbols are used to represent vectors. 
The energy balance in the fluid phase is given by

$$
\rho_{f} C p_{f}\left(\frac{\partial T_{f}}{\partial t}+u(r) \frac{\partial T_{f}}{\partial x}\right)=k_{f} \nabla_{\perp}^{2} T_{f}+k_{f} \frac{\partial^{2} T_{f}}{\partial x^{2}} \text { in } \Omega_{f}
$$

The energy balance in the washcoat is

$$
\rho_{w c} C p_{w c} \frac{\partial T_{w c}}{\partial t}=k_{w c} \nabla_{\perp}^{2} T_{w c}+k_{w c} \frac{\partial^{2} T_{w c}}{\partial x^{2}}+(-\Delta H) R\left(C_{w c}, T_{w c}\right) \text { in } \Omega_{w c}
$$

and the energy balance in the substrate can be expressed as

$$
\rho_{w} C p_{w} \frac{\partial T_{w}}{\partial t}=k_{w} \nabla_{\perp}^{2} T_{w}+k_{w} \frac{\partial^{2} T_{w}}{\partial x^{2}} \text { in } \Omega_{w}
$$

Here, $T$ represents temperature, $\rho$ and $C p$ are density and specific heat capacity, and $k$ is thermal conductivity. The subscript $f$ stands for the fluid phase, $w c$ and $w$ represent the washcoat and the substrate wall, respectively. $\Delta H$ is the heat of reaction. The initial and boundary conditions for the energy balances are given by

$$
\begin{gathered}
T_{f}=T_{f}^{0}(x, r), T_{w c}=T_{w c}^{0}(x, r), T_{w}=T_{w}^{0}(x, r) \text { at } t=0 \\
k_{f} \mathbf{n}_{\Omega_{f}} \cdot \nabla_{\perp} T_{f}=k_{w c} \mathbf{n}_{\Omega_{f}} \cdot \nabla_{\perp} T_{w c}, T_{f}=T_{w c} \text { on } \partial \Omega_{f}, \\
k_{w c} \mathbf{n}_{\Omega_{w c}} \cdot \nabla_{\perp} T_{w c}=k_{w} \mathbf{n}_{\Omega_{w c}} \cdot \nabla_{\perp} T_{w}, T_{w c}=T_{w} \text { on } \partial \Omega_{w c}, \\
\frac{\mathbf{n}_{\Omega_{w}} \cdot \nabla_{\perp} T_{w}=0 \text { on } \partial \Omega_{w},}{\rho_{f} C p_{f}} \frac{\partial T_{f}}{\partial x}=u\left(T_{f}-T_{f}^{i n}(t)\right), \frac{\partial T_{w c}}{\partial x}=\frac{\partial T_{w}}{\partial x}=0 \text { at } x=0, \\
\frac{\partial T_{f}}{\partial x}=\frac{\partial T_{w c}}{\partial x}=\frac{\partial T_{w}}{\partial x}=0 \text { at } x=L .
\end{gathered}
$$

Here, $\mathbf{n}_{\Omega_{w}}$ is the unit normal vector radially outward to the substrate boundary $\partial \Omega_{w}\left(r=2 R_{\Omega}+\delta_{w c}+\right.$ $\left.\delta_{w}\right) ; \delta_{w c}$ is the thickness of the washcoat and $\delta_{w}$ is half-thickness of the substrate wall. [Remark: The boundary conditions for the fluid phase in Eqs. 3d,3e,7e and $7 f$ represent the so called "Danckwerts boundary conditions" [3].] 
Table 1: Characteristic scales

\begin{tabular}{lll}
\hline Scale & Definition & Description \\
\hline Time & $\tau=L / \bar{u}$ & Space time $(\mathrm{s})$ \\
Axial length & $L$ & Reactor length $(\mathrm{m})$ \\
Transverse length & $R_{\Omega}$ & Hydraulic radius $(\mathrm{m})$ \\
Concentration & $\bar{C}_{f}^{i n}$ & Inlet concentration $\left(\mathrm{mol} \cdot \mathrm{m}^{-3}\right)$ \\
Temperature & $\Delta T_{a d}=\frac{(-\Delta H) \bar{C}_{f}^{i n}}{\rho_{f} C p_{f}}$ & Adiabatic temperature rise $(\mathrm{K})$ \\
\hline
\end{tabular}

Cycling experiments are commonly conducted to evaluate after-treatment catalyst performance, where inlet concentrations and/or fluid temperature are varied periodically. In such cases, the inlet conditions $\left(C_{f}^{i n}\right.$ and $\left.T_{f}^{i n}\right)$ are periodic functions of time. Here, we take sinusoidal functions as an example,

$$
\begin{aligned}
& C_{f}^{i n}(t)=\bar{C}_{f}^{i n}\left(1+\varepsilon_{C} \sin (2 \pi \omega t)\right), \\
& T_{f}^{i n}(t)=\bar{T}_{f}^{i n}\left(1+\varepsilon_{T} \sin (2 \pi \omega t)\right),
\end{aligned}
$$

where $\bar{C}_{f}^{i n}$ and $\bar{T}_{f}^{i n}$ are cycle averaged values of inlet concentration and fluid temperature; $\varepsilon_{C}$ and $\varepsilon_{T}$ are relative cycling amplitude of inlet concentration and fluid temperature, respectively; $\omega$ is the cycling frequency which is usually the same for both inlet concentration and fluid temperature. For steady-state experiments, $\varepsilon_{C}=\varepsilon_{T}=0, C_{f}^{i n}=\bar{C}_{f}^{i n}, T_{f}^{i n}=\bar{T}_{f}^{i n}$.

\subsection{Dimensionless groups}

As stated in the introduction, scale-up problems are usually studied by comparing various dimensionless groups. If two reactors have all dimensionless groups matched, the mathematical models will become identical indicating that the performances of these two reactors will also be identical. We define characteristic scales as shown in Table 1, then non-dimensionalized the variables, operators and inlet conditions accordingly,

$$
\widehat{t}=\frac{t}{\tau}, z=\frac{x}{L}, \xi=\frac{r}{R_{\Omega}}, y=\frac{C}{\bar{C}_{f}^{i n}}, \widehat{T}=\frac{T-\bar{T}_{f}^{i n}}{\Delta T_{a d}}, \theta^{i n}=\frac{\bar{T}_{f}^{i n}}{\Delta T_{a d}}, \widehat{\nabla}_{\perp}=R_{\Omega} \nabla_{\perp}, \widehat{\nabla}_{\perp}^{2}=R_{\Omega}^{2} \nabla_{\perp}^{2}
$$

Notice that instead of introducing a predefined reference temperature, we use the adiabatic temperature rise as the temperature scale. In this way, the dimensionless temperature rise $\widehat{T}$ has the same 
order of magnitude as the conversion $\chi=1-y$. The adiabatic temperature rise can also be rewritten as

$$
\Delta T_{a d}=\frac{(-\Delta H) \bar{C}_{f}^{i n}}{\rho_{f} C p_{f}}=\frac{(-\Delta H) \bar{X}_{f}^{i n}}{\widehat{C p}_{f}}
$$

where $\widehat{C p}_{f}$ is the molar heat capacity of the fluid which can be assumed constant for dilute reactant, $\bar{X}_{f}^{i n}$ is the average inlet mole fraction of the limiting reactant. For a given reaction, the adiabatic temperature rise is proportional to $\bar{X}_{f}^{i n}$, so all results involving $\Delta T_{a d}$ can also be interpreted in terms of $\bar{X}_{f}^{i n}$ in the following sections. Substituting the dimensionless variables in the equations, some important dimensionless groups appear in the governing equations and inlet/boundary conditions. The model equations may be expressed as

$$
\begin{gathered}
\frac{\partial y_{f}}{\partial \widehat{t}}+2\left(1-\frac{1}{4} \xi^{2}\right) \frac{\partial y_{f}}{\partial z}=\frac{1}{P_{m, f}} \widehat{\nabla}_{\perp}^{2} y_{f}+\frac{1}{P e_{m, f}} \frac{\partial^{2} y_{f}}{\partial z^{2}} \\
\epsilon^{\prime} \frac{\partial y_{w c}}{\partial \widehat{t}}=\frac{1}{P_{m, w c}} \widehat{\nabla}_{\perp}^{2} y_{w c}+\frac{1}{P e_{m, w c}^{\prime}} \frac{\partial^{2} y_{w c}}{\partial z^{2}}-D a_{0}^{\prime} y_{w c} \exp \left(\frac{-1 / \beta}{\widehat{T}_{w c}+\theta^{i n}}\right) \\
\frac{\partial \widehat{T}_{f}}{\partial \widehat{t}}+2\left(1-\frac{1}{4} \xi^{2}\right) \frac{\partial \widehat{T}_{f}}{\partial z}=\frac{1}{P_{h, f}} \widehat{\nabla}_{\perp}^{2} \widehat{T}_{f}+\frac{1}{P e_{h, f}} \frac{\partial^{2} \widehat{T}_{f}}{\partial z^{2}} \\
\frac{\partial \widehat{T}_{w c}}{\partial \widehat{t}}=\frac{1}{P_{h, w c}} \widehat{\nabla}_{\perp}^{2} \widehat{T}_{w c}+\frac{1}{P e_{h, w c}^{\prime}} \frac{\partial^{2} \widehat{T}_{w c}}{\partial z^{2}}+D a_{0}^{\prime} y_{w c} \exp \left(\frac{-1 / \beta}{\widehat{T}_{w c}+\theta^{i n}}\right) \\
\sigma_{w} \frac{\partial \widehat{T}_{w}}{\partial \hat{t}}=\frac{1}{P_{h, w}} \widehat{\nabla}_{\perp}^{2} \widehat{T}_{w}+\frac{1}{P e_{h, w}^{\prime}} \frac{\partial^{2} \widehat{T}_{w}}{\partial z^{2}}
\end{gathered}
$$

with initial and boundary conditions,

$$
\begin{gathered}
y_{f}=y_{f}^{0}(z, \xi), y_{w c}=y_{w c}^{0}(z, \xi) \text { at } \widehat{t}=0, \\
\widehat{T}_{f}=\widehat{T}_{f}^{0}(z, \xi), \widehat{T}_{w c}=\widehat{T}_{w c}^{0}(z, \xi), \widehat{T}_{w}=\widehat{T}_{w}^{0}(z, \xi) \text { at } \widehat{t}=0, \\
\frac{1}{P_{m, f}} \mathbf{n}_{\Omega_{f}} \cdot \widehat{\nabla}_{\perp} y_{f}=\frac{1}{P_{m, w c}} \mathbf{n}_{\Omega_{f}} \cdot \widehat{\nabla}_{\perp} y_{w c}, y_{f}=y_{w c} \text { at } \xi=2, \\
\mathbf{n}_{\Omega_{w c}} \cdot \widehat{\nabla}_{\perp} y_{w c}=0 \text { at } \xi=2+\frac{\delta_{w c}}{R_{\Omega}}, \\
\frac{1}{P e_{m, f}} \frac{\partial y_{f}}{\partial z}=y_{f}-y_{f}^{i n}(\widehat{t}), \frac{\partial y_{w c}}{\partial z}=0 \text { at } z=0,
\end{gathered}
$$




$$
\begin{gathered}
\frac{\partial y_{f}}{\partial z}=\frac{\partial y_{w c}}{\partial z}=0 \text { at } z=1 . \\
\frac{1}{P_{h, f}} \mathbf{n}_{\Omega_{f}} \cdot \widehat{\nabla}_{\perp} \widehat{T}_{f}=\frac{1}{P_{h, w c}} \mathbf{n}_{\Omega_{f}} \cdot \widehat{\nabla}_{\perp} \widehat{T}_{w c}, \widehat{T}_{f}=\widehat{T}_{w c} \text { at } \xi=2 \\
\frac{1}{P_{h, w c}} \mathbf{n}_{\Omega_{w c}} \cdot \widehat{\nabla}_{\perp} \widehat{T}_{w c}=\frac{1}{P_{h, w}} \mathbf{n}_{\Omega_{w c}} \cdot \widehat{\nabla}_{\perp} \widehat{T}_{w}, \widehat{T}_{w c}=\widehat{T}_{w} \text { at } \xi=2+\frac{\delta_{w c}}{R_{\Omega}} \\
\frac{1}{P e_{h, f}} \frac{\partial \widehat{T}_{f}}{\partial z}=\widehat{T}_{f}-\widehat{\nabla}_{\perp} \widehat{T}_{w}^{i n}=0 \text { at } \xi=2+\frac{\partial \hat{T}_{w c}+\delta_{w}}{\partial z}=\frac{\partial \widehat{T}_{w}}{\partial z}=0 \text { at } z=0 \\
\frac{\partial \widehat{T}_{f}}{\partial z}=\frac{\partial \widehat{T}_{w c}}{\partial z}=\frac{\partial \widehat{T}_{w}}{\partial z}=0 \text { at } z=1
\end{gathered}
$$

For periodic inlet forcing, the dimensionless inlet concentration and fluid temperature are

$$
y_{f}^{i n}(\hat{t})=1+\varepsilon_{C} \sin (2 \pi \widehat{\omega} \hat{t}), \widehat{T}_{f}^{i n}(\hat{t})=\theta^{i n} \varepsilon_{T} \sin (2 \pi \widehat{\omega} \hat{t}),
$$

where the dimensionless cycling frequency $\widehat{\omega}=\omega \tau$. The capacitance parameters are defined by

$$
\epsilon^{\prime}=\epsilon_{w c}, \sigma_{w c}=\frac{\rho_{w c} C p_{w c}}{\rho_{f} C p_{f}}, \sigma_{w}=\frac{\rho_{w} C p_{w}}{\rho_{f} C p_{f}}
$$

The various transverse Péclet numbers (related to species and heat diffusion in the transverse direction) are defined by

$$
\begin{gathered}
P_{m, f}=\frac{\bar{u} R_{\Omega}^{2}}{D_{f} L}, P_{m, w c}=\frac{\bar{u} R_{\Omega}^{2}}{D_{w c} L}, \\
P_{h, f}=\frac{\bar{u} R_{\Omega}^{2}}{\alpha_{f} L}, P_{h, w c}=\frac{\bar{u} R_{\Omega}^{2}}{\alpha_{f} L} \frac{k_{f}}{k_{w c}}, P_{h, w}=\frac{\bar{u} R_{\Omega}^{2}}{\alpha_{f} L} \frac{k_{f}}{k_{w}},
\end{gathered}
$$

where, $\alpha_{f}$ is the thermal diffusivity of the fluid defined by

$$
\alpha_{f}=\frac{k_{f}}{\rho_{f} C p_{f}}
$$

The various axial Péclet numbers (related to heat and mass diffusion in the flow direction, washcoat and wall) are defined by

$$
P e_{m, f}=\frac{\bar{u} L}{D_{f}}, P e_{m, w c}^{\prime}=\frac{\bar{u} L}{D_{w c}}
$$




$$
P e_{h, f}=\frac{\bar{u} L}{\alpha_{f}}, P e_{h, w c}^{\prime}=\frac{\bar{u} L}{\alpha_{f}} \frac{k_{f}}{k_{w c}}, P e_{h, w}^{\prime}=\frac{\bar{u} L}{\alpha_{f}} \frac{k_{f}}{k_{w}} .
$$

The subscripts $m$ and $h$ represent mass and heat transfer, $f, w c$ and $w$ represent the flow channel, the washcoat and the substrate wall, respectively. For a first-order reaction with rate expression of the form $R=A \exp \left(-\frac{E_{a}}{R_{g} T}\right) C$, the Damköhler number is defined as $D a_{0}^{\prime}=A \tau$, where $A$ is the pre-exponential factor in the Arrhenius equation. The dimensionless adiabatic temperature rise $\beta$ is defined as

$$
\beta=\frac{\Delta T_{a d} R_{g}}{E_{a}}
$$

where $E_{a}$ is the activation energy of the reaction, $R_{g}$ is the gas constant. In the following sections, due to radial averaging, the definitions of some dimensionless groups will be different. The groups appearing in this detailed model are denoted by a prime symbol to distinguish them from those used in the simplified models.

In this work, because the dependence of transport coefficients on temperature does not qualitatively affect the comparison of reactors, we assume that they are constants for simplicity. To apply the criteria discussed in this work, one should evaluate the transport coefficients at the temperature of interest (e.g. ignition temperature) or use average values over the temperature range of interest.

We note that the model has 21 dimensionless groups consisting of cycling amplitudes and frequency, various capacitance ratios, geometric ratios, reaction parameters and Péclet numbers. Among all the dimensionless groups, the ones without reactor length $L$, or with only the ratio of $L / \bar{u}$ (space time $\tau$ ), including $\varepsilon_{C}, \varepsilon_{T}, \widehat{\omega}, \epsilon^{\prime}, \sigma_{w c}, \sigma_{w}, \frac{\delta_{w}}{R_{\Omega}}, \frac{\delta_{w c}}{R_{\Omega}}, D a_{0}^{\prime}, \theta^{i n}, \beta$, and the five transverse Péclet numbers remain exactly the same in both lab-scale and full-scale reactors [Remark: The parameter $\beta$ remains the same in both scales only if the same limiting reactant concentrations are used]. However, in the five axial Péclet numbers, the reactor length $L$ and gas velocity $\bar{u}$ appear as a product. Thus, although the same space time is maintained in different scales, the axial Péclet numbers vary quadratically with reactor length (or scale factor). The discrepancy of the axial Péclet numbers is one main reason for most of the scale-up issues discussed in this work. Although it is not possible to match the axial Péclet numbers in different scales, the impact of them (axial dispersion/conduction) can be neglected when they are very large and the adiabatic temperature rise is small. In that case, all the $\frac{1}{P e} \frac{\partial^{2}}{\partial z^{2}}$ terms will be negligible, resulting in a two-dimensional (parabolic) model (with plug 
flow in the axial direction but with radial heat and mass diffusion included). In this case, similarity can be found in reactors of different scales. However, this is rarely satisfied since the axial Péclet numbers in the lab-scale reactors are usually small (typically by a factor 10 to 100 compared to the full-scale systems).

\subsection{Simplified Models}

In catalytic after-treatment reactors, the transverse Péclet numbers are usually very small, indicating that the concentration and temperature variations across the channel are small. Thus, it is reasonable to use radially lumped models to analyze scale-up issues. Although this assumption of small radial gradients and radial averaging may introduce small error, it may be justified for two reasons: (i) as noted above, it is the axial Péclet numbers that do not match at different scales (ii) models of different scales will be influenced in the same way since all the transverse Péclet numbers are exactly matched.

\subsubsection{One-dimensional two-phase monolith model}

When radial gradients are small, a one-dimensional two-phase model is commonly used. Radially averaging the two-dimensional model and using mass and heat transfer coefficients [6], the model becomes one-dimensional. Thus, the species balance in the fluid phase is simplified to

$$
\frac{\partial y_{f}}{\partial \widehat{t}}+\frac{\partial y_{f}}{\partial z}=\frac{1}{P e_{m, f}} \frac{\partial^{2} y_{f}}{\partial z^{2}}-\frac{1}{P_{m}}\left(y_{f}-y_{w c}\right)
$$

while the species balance in the washcoat is given by

$$
\epsilon \frac{\partial y_{w c}}{\partial \widehat{t}}=\frac{1}{P e_{m, w c}} \frac{\partial^{2} y_{w c}}{\partial z^{2}}+\frac{1}{P_{m}}\left(y_{f}-y_{w c}\right)-D a_{0} y_{w c} \exp \left(\frac{-1 / \beta}{\widehat{T}_{s}+\theta^{i n}}\right) .
$$

For simplicity, we keep the same notation for the state variables. However, unlike in the twodimensional case where they are functions of the radial position, in the one-dimensional two-phase model they are considered radially averaged values. For example, $y_{f}$ and $y_{w c}$, represent the cupmixing (dimensionless) concentration in the fluid phase and the volume averaged (dimensionless) 
concentration in the washcoat, respectively. Because of the radial averaging, some geometric factors appear in the lumped parameters:

$$
\epsilon=\epsilon^{\prime} \frac{\delta_{w c}}{R_{\Omega}}, D a_{0}=D a_{0}^{\prime} \frac{\delta_{w c}}{R_{\Omega}}, P e_{m, w c}=P e_{m, w c}^{\prime} \frac{R_{\Omega}}{\delta_{w c}}
$$

Further, $P_{m, f}$ and $P_{m, w c}$ are lumped into an overall transverse mass Péclet number $P_{m}$ defined by

$$
P_{m}=P_{m, f} \frac{4}{S h_{e}}+P_{m, w c} \frac{\delta_{w c}}{R_{\Omega}} \frac{1}{S h_{i}}
$$

where $S h_{e}$ and $S h_{i}$ are external and internal Sherwood numbers, respectively. The Sherwood numbers are defined as $[7]$

$$
S h_{e}=\frac{4 k_{m, e} R_{\Omega}}{D_{f}}, S h_{i}=\frac{k_{m, i} R_{\Omega}}{D_{w c}},
$$

where $k_{m, e}$ and $k_{m, i}$ are external and internal mass transfer coefficients.

The energy balance in the fluid phase is

$$
\frac{\partial \widehat{T}_{f}}{\partial \widehat{t}}+\frac{\partial \widehat{T}_{f}}{\partial z}=\frac{1}{P e_{h, f}} \frac{\partial^{2} \widehat{T}_{f}}{\partial z^{2}}-\frac{1}{P_{h}}\left(\widehat{T}_{f}-\widehat{T}_{s}\right)
$$

Since the thermal conductivities of the washcoat and the substrate wall are both large and the thicknesses are small, in the energy balance we assume the temperature is radially uniform in the whole solid phase including both the washcoat and the substrate wall. The parameters used for the solid phase can be defined as an effective or averaged value for the two layers,

$$
\begin{gathered}
\delta_{s}=\delta_{w}+\delta_{w c}, \delta_{s} k_{s}=\delta_{w} k_{w}+\delta_{w c} k_{w c} \\
\rho_{s} C p_{s} \delta_{s}=\rho_{w c} C p_{w c} \delta_{w c}+\rho_{w} C p_{w} \delta_{w} \\
P_{h, s}=\frac{\bar{u} R_{\Omega}^{2}}{\alpha_{f} L} \frac{k_{f}}{k_{s}}=\frac{\delta_{w c}+\delta_{w}}{\frac{\delta_{w c}}{P_{h, w c}}+\frac{\delta_{w}}{P_{h, w}}} \\
P e_{h, s}=\frac{\bar{u} L}{\alpha_{f}} \frac{k_{f}}{k_{s}} \frac{R_{\Omega}}{\delta_{s}}=\frac{1}{\frac{1}{P e_{h, w c}^{\prime}} \frac{\delta_{w c}}{R_{\Omega}}+\frac{1}{P e_{h, w}^{\prime}} \frac{\delta_{w}}{R_{\Omega}}}
\end{gathered}
$$

where the subscript $s$ stands for the solid phase including both the washcoat and the substrate. The 
energy balance in the solid phase can be expressed as

$$
\sigma_{s} \frac{\partial \widehat{T}_{s}}{\partial \widehat{t}}=\frac{1}{P_{h}}\left(\widehat{T}_{f}-\widehat{T}_{s}\right)+\frac{1}{P e_{h, s}} \frac{\partial^{2} \widehat{T}_{s}}{\partial z^{2}}+D a_{0} y_{w c} \exp \left(\frac{-1 / \beta}{\widehat{T}_{s}+\theta^{i n}}\right)
$$

The heat capacity ratio $\sigma_{s}$ is defined as

$$
\sigma_{s}=\frac{\rho_{s} C p_{s} \delta_{s}}{\rho_{f} C p_{f} R_{\Omega}}
$$

$P_{h, f}$ and $P_{h, s}$ are lumped into an overall transverse heat Péclet number $P_{h}$ as

$$
P_{h}=P_{h, f} \frac{4}{N u_{e}}+P_{h, s} \frac{\delta_{s}}{R_{\Omega}} \frac{1}{N u_{i}}
$$

where $N u_{e}$ and $N u_{i}$ are external and internal Nusselt numbers, respectively. The Nusselt numbers are defined as

$$
N u_{e}=\frac{4 h_{e} R_{\Omega}}{k_{f}}, N u_{i}=\frac{h_{i} R_{\Omega}}{k_{s}}
$$

where $h_{e}$ and $h_{i}$ are external and internal heat transfer coefficients.

The initial and boundary conditions for this one-dimensional two-phase model are given by

$$
\begin{gathered}
y_{f}=y_{f}^{0}(z), y_{w c}=y_{w c}^{0}(z) \text { at } \widehat{t}=0, \\
\widehat{T}_{f}=\widehat{T}_{f}^{0}(z), \widehat{T}_{s}=\widehat{T}_{s}^{0}(z) \text { at } \widehat{t}=0, \\
\frac{1}{P e_{m, f}} \frac{\partial y_{f}}{\partial z}=y_{f}-y_{f}^{i n}(\widehat{t}), \frac{\partial y_{w c}}{\partial z}=0 \text { at } z=0, \\
\frac{\partial y_{f}}{\partial z}=\frac{\partial y_{w c}}{\partial z}=0 \text { at } z=1, \\
\frac{\partial \widehat{T}_{f}}{P e_{h, f}}=\widehat{T}_{f}-\widehat{T}_{f}^{i n}(\widehat{t}), \frac{\partial \widehat{T}_{s}}{\partial z}=0 \text { at } z=0, \\
\frac{\partial \widehat{T}_{f}}{\partial z}=\frac{\partial \widehat{T}_{s}}{\partial z}=0 \text { at } z=1 .
\end{gathered}
$$

Since the thermal conductivity in the solid phase is much larger than that in the fluid phase, 
$P_{h, s} \ll P_{h, f}$, thus

$$
P_{h} \approx P_{h, f} \frac{4}{N u_{e}}=\frac{\bar{u} R_{\Omega}^{2}}{\alpha_{f} L} \frac{4}{N u_{e}},
$$

and the term containing the internal Nusselt number $\left(N u_{i}\right)$ can usually be neglected. Gundlapally and Balakotaiah [8] showed that $N u_{e}$ and $S h_{e}$ are functions of space time, radial geometric parameters and diffusivities of the species, which are either independent of $L$ or dependent on the ratio $L / \bar{u}$. Kumar et al. $[9,10]$ provided a correlation for $S h_{i}$, which involves only kinetic parameters and local properties. Thus, the transverse heat and mass Péclet numbers $\left(P_{h}\right.$ and $\left.P_{m}\right)$ are matched exactly in reactors with different scales provided that the same space time is maintained. This confirms our conclusion in Section 2.2 that the radial mass and heat transfer is matched in lab-scale and full-scale reactors. Although this one-dimensional two-phase model has fewer dimensionless groups due to radial lumping, all information from Section 2.2 is retained. The dimensionless groups from the two-dimensional model are either kept the same (e.g. $\left.P e_{m, f}\right)$ or lumped with similar group to form a combined group (e.g. $P_{m}$ ). [Remark: Radial lumping has reduced the 21 dimensionless groups (5 axial Péclet numbers) of the two-dimensional model into 14 dimensionless groups (4 axial Péclet numbers) of the one-dimensional two-phase model.]

\subsubsection{Pseudo-homogeneous model}

If the lumped transverse Péclet numbers $P_{m}$ and $P_{h}$ are very small, one can combine separate phase balances and form a pseudo-homogeneous model by utilizing effective Péclet number $P e_{e f f}$ defined as $[11]$

$$
\frac{1}{P e_{e f f}}=\frac{1}{P e}+P,
$$

which combines the effects of axial dispersion and interphase (radial) gradient. Here, for simplicity, we examine this homogeneous limit, i.e. $P_{m}=P_{h}=0$. As mentioned in Section 2.3, since the transverse Péclet numbers are exactly matched, and they are small, neglecting their effects will not qualitatively effect our analysis. The impact of this simplification is further discussed in Section 5.

When combining separate phase balances, the axial Péclet numbers for the pseudo-homogeneous model, $P e_{m}$ and $P e_{h}$, are defined as 


$$
\begin{gathered}
P e_{m}=\frac{1}{\frac{1}{P e_{m, f}}+\frac{1}{P e_{m, w c}}}=\frac{\bar{u} L}{D_{f}} \frac{1}{1+\frac{D_{w c}}{D_{f}} \frac{\delta_{w c}}{R_{\Omega}}} \approx P e_{m, f}, \\
P e_{h}=\frac{1}{\frac{1}{P e_{h, f}}+\frac{1}{P e_{h, s}}}=\frac{\bar{u} L}{\alpha_{f}} \frac{k_{f}}{k_{s}} \frac{R_{\Omega}}{\delta_{s}} \frac{1}{\frac{k_{f}}{k_{s}} \frac{R_{\Omega}}{\delta_{s}}+1} \approx P e_{h, s} .
\end{gathered}
$$

Thus, when the axial Péclet number in one phase is significantly larger than the one in the other phase, the smaller one will dominate the behavior of the pseudo-homogeneous model. In the case of mass transfer, since the molecular diffusivity in the washcoat $\left(D_{w c}\right)$ can be two to three orders of magnitude smaller than the molecular diffusivity in the fluid phase, $P e_{m, f}$ is usually much smaller than $P e_{m, s}$. Thus, it is reasonable to assume $P e_{m} \approx P e_{m, f}$. Similarly in the case of axial heat diffusion, $P e_{h, f}$ is usually much larger than $P e_{h, s}$ (as the solid thermal conductivity is about two orders of magnitude larger than that of the fluid). Assuming $P e_{h} \approx P e_{h, s}$ will only lead to small error.

Here, we examine the ratio $P e_{m} / P e_{h}$,

$$
\frac{P e_{m}}{P e_{h}} \approx \frac{P e_{m, f}}{P e_{h, s}}=\frac{k_{s}}{k_{f}} \frac{\delta_{s}}{R_{\Omega}} L e_{f}
$$

where

$$
L e_{f}=\frac{\alpha_{f}}{D_{f}}
$$

is the Lewis number depending only on the physical properties of the gas and diffusivity of the limiting reactant species. Further, $\delta_{s} / R_{\Omega}$ is a constant depending on the monolith channel structure, and $k_{s} / k_{f}$ depends on the material of the monolith and thermal conductivity of the fluid. Thus, under the assumptions of this work, the $P e_{m} / P e_{h}$ ratio remains the same regardless of the reactor length. For ceramic monolith, this ratio is about 10 while for metallic monolith the ratio can be 100 or up to several hundreds.

With the dimensional analysis and simplifications, we arrive at a monolith model that is simple yet sufficient to explore the differences in reactors of different scales. For convenience and symmetry of the equations, we use the conversion $\chi=1-y$ instead of the dimensionless concentration $y$, in the species balance, 


$$
(1+\epsilon) \frac{\partial \chi}{\partial \widehat{t}}=\frac{1}{P e_{m}} \frac{\partial^{2} \chi}{\partial z^{2}}-\frac{\partial \chi}{\partial z}+D a_{0}(1-\chi) \exp \left(\frac{-1 / \beta}{\widehat{T}+\theta^{i n}}\right)
$$

The energy balance is

$$
\left(1+\sigma_{s}\right) \frac{\partial \widehat{T}}{\partial \widehat{t}}=\frac{1}{P e_{h}} \frac{\partial^{2} \widehat{T}}{\partial z^{2}}-\frac{\partial \widehat{T}}{\partial z}+D a_{0}(1-\chi) \exp \left(\frac{-1 / \beta}{\widehat{T}+\theta^{i n}}\right)
$$

The boundary conditions are

$$
\begin{gathered}
\frac{1}{P e_{m}} \frac{\partial \chi}{\partial z}-\chi=y_{f}^{i n}(\hat{t})-1=\varepsilon_{C} \sin (2 \pi \widehat{\omega} \widehat{t}), \text { at } z=0 \\
\frac{1}{P e_{h}} \frac{\partial \widehat{T}}{\partial z}-\widehat{T}=-\widehat{T}_{f}^{i n}(\hat{t})=-\theta^{i n} \varepsilon_{T} \sin (2 \pi \widehat{\omega} \widehat{t}), \text { at } z=0, \\
\frac{\partial \chi}{\partial z}=\frac{\partial \widehat{T}}{\partial z}=0 \text { at } z=1 .
\end{gathered}
$$

[Remark: In simplifying the one-dimensional two-phase model further to this pseudo-homogeneous model, we have reduced the number of dimensionless groups to 10 and the axial Péclet numbers to 2. The steady-state version of this model is characterized by five groups, i.e. $D a_{0}, \beta, \theta^{i n}, P e_{m}$ and $\left.P e_{h}.\right]$ This model has three limiting cases of significance. When both $P e_{m} \rightarrow 0$ and $P e_{h} \rightarrow 0$, the model approaches a continuous-flow stirred tank reactor (CSTR) asymptote; when $P e_{m} \rightarrow \infty$ and $P e_{h} \rightarrow \infty$, the model approaches a plug flow reactor (PFR) asymptote; when $P e_{m} \rightarrow \infty$ and $P e_{h} \rightarrow 0$, the model becomes a lumped thermal reactor model (LTR). Because $P e_{m}$ is usually larger than $P e_{h}$, the asymptote where $P e_{m} \rightarrow 0$ and $P e_{h} \rightarrow \infty$ is not important from a practical point of view.

Since the dimensionless time $\widehat{t}$ and frequency $\widehat{\omega}$ contain only the ratio of $L / \bar{u}$, which is matched in different scales, the transient performances of lab- and full-scale reactors will be similar provided that their steady-state performances (e.g. light-off curves, hysteresis behaviors) are similar. Thus, in the following sections, we consider mainly the steady-state versions of the simplified models to study the scale-up issues. In the steady-state models, all $\widehat{t}$ containing terms are neglected and the equations become nonlinear ordinary differential equations (ODEs) in the axial coordinate. 


\section{Impact of mass dispersion}

As discussed above, the mass Péclet number $P e_{m}$ is usually much larger than heat Péclet number $P e_{h}$, the ratio can vary from 10 (for ceramic monolith) to 100 (for metallic monolith). Thus, $P e_{m}$ usually has less impact on the reactor behavior than $P e_{h}$ in most non-isothermal operations and one can often neglect the axial diffusion term in the species balance. However, in isothermal operations, different $P e_{m}$ (different reactor length scales) can also lead to significant quantitative and sometimes qualitative differences.

\subsection{Linear kinetics}

In case of an isothermal reactor, where $\Delta T_{a d}=0, T=T^{i n}$, for a first-order reaction the steady-state pseudo-homogeneous model becomes

$$
\begin{gathered}
\frac{1}{P e_{m}} \frac{d^{2} \chi}{d z^{2}}-\frac{d \chi}{d z}+D a_{0}(1-\chi) \exp \left(-\frac{E_{a}}{R T^{\text {in }}}\right)=0 \\
\left.\frac{1}{P e_{m}} \frac{d \chi}{d z}\right|_{z=0}-\chi(z=0)=0,\left.\frac{d \chi}{d z}\right|_{z=1}=0
\end{gathered}
$$

The solution of the above isothermal axial dispersion model can be found in chemical engineering textbooks $[12,13]$. The exit conversion is given by

$$
\chi^{e x i t}=1-\frac{4 q \exp \left(P e_{m} / 2\right)}{(1+q)^{2} \exp \left(q P e_{m} / 2\right)-(1-q)^{2} \exp \left(-q P e_{m} / 2\right)},
$$

where

$$
q=\sqrt{1+\frac{4 D a_{0}}{P e_{m}} \exp \left(-\frac{E_{a}}{R T^{i n}}\right)}
$$

The two asymptotic cases where $P e_{m} \rightarrow \infty(\mathrm{PFR})$ and $P e_{m} \rightarrow 0$ (CSTR) can also be solved as

$$
\begin{gathered}
\chi_{P F R}^{e x i t}=1-\exp \left(-D a_{0} \exp \left(-\frac{E_{a}}{R T^{i n}}\right)\right), \\
\chi_{C S T R}^{\text {exit }}=1-\frac{1}{1+D a_{0} \exp \left(-\frac{E_{a}}{R T^{i n}}\right)} .
\end{gathered}
$$




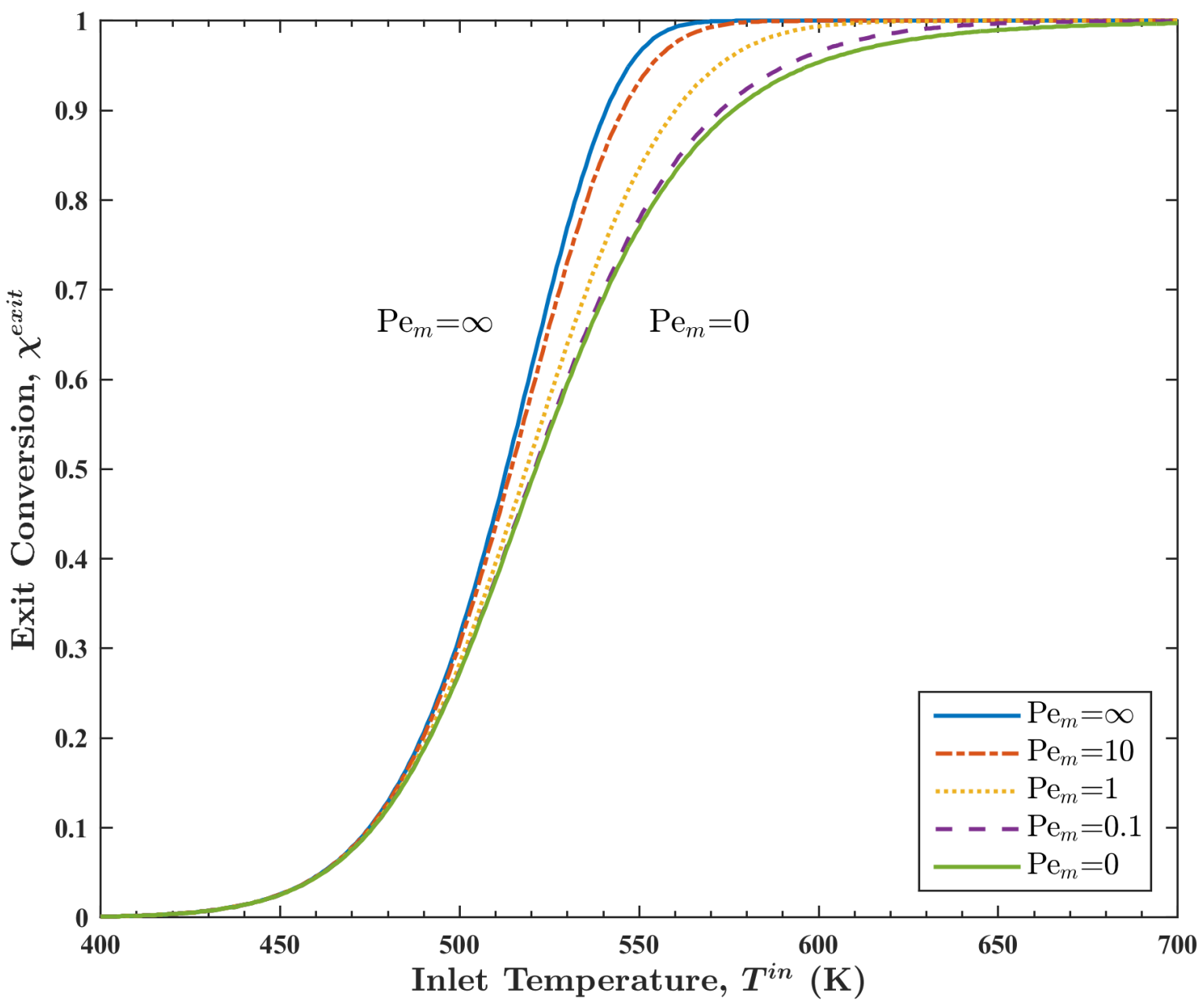

Figure 3: Plot of steady-state exit conversion versus inlet temperature in isothermal reactors with first-order kinetics and different axial mass Péclet numbers $\left(P e_{m}\right) .\left(E_{a}=100 \mathrm{~kJ} / \mathrm{mol}, D a_{0}=10^{10}\right)$

Shown in Fig. 3 are steady-state conversions of isothermal reactors with different $P e_{m}$, where the kinetic parameters are chosen to be typical values of after-treatment catalysts $\left(E_{a}=100 \mathrm{~kJ} / \mathrm{mol}\right.$, $\left.D a_{0}=10^{10}\right)$. When $P e_{m}$ is smaller than 10 , mass dispersion can introduce significant quantitative errors especially at high conversions $\left(\chi^{\text {exit }}>0.8\right.$ ), which is the range of interest. In that case, direct scale-up according to lab-scale performance will result in an overdesign of the catalyst loading for full-scale reactors.

\section{$3.2 \quad$ Nonlinear kinetics}

When the kinetics are nonlinear, mass dispersion can create multiple solutions in the steady-state conversions. Hence, the difference between reactors with different $P e_{m}$ can be qualitative. LangmuirHinshelwood mechanism and Voltz kinetics are widely accepted as global kinetic models for $C O$ 
oxidation on $\mathrm{Pt}$ and other oxidation reactions in after-treatment catalysts [14-16]. Here we take Langmuir-Hinshelwood kinetics for $C O$ oxidation as an example, and the rate expression as

$$
R=\frac{A \exp \left(-\frac{E_{a}}{R T}\right) C_{O_{2}} C_{C O}}{\left(1+K_{a d s} C_{C O}\right)^{2}},
$$

where $K_{a d s}$ is the adsorption equilibrium constant. When the concentration of $C O$ is high, the reaction is inhibited by $C O$ and close to negative first-order with respect to $C O$.

We assume that $\mathrm{O}_{2}$ is in large excess so that $\mathrm{C}_{\mathrm{O}_{2}}$ can be treated as a constant and can be lumped into the rate constant. The dimensionless adsorption equilibrium constant $\kappa$ is defined as

$$
\kappa=K_{a d s} C_{t o t a l},
$$

where $C_{\text {total }}$ is total fluid concentration. Then, the Damköhler number can be defined as

$$
D a_{0}^{L H}=\frac{A \tau C_{O_{2}}}{\left(1+\kappa X_{C O}^{i n}\right)^{2}} \frac{\delta_{w c}}{R_{\Omega}} .
$$

The model equations in terms of $C O$ conversion are

$$
\begin{gathered}
\frac{1}{P e_{m}} \frac{d^{2} \chi}{d z^{2}}-\frac{d \chi}{d z}+D a_{0}^{L H} \exp \left(-\frac{E_{a}}{R T^{i n}}\right) \frac{\left(1+\kappa X_{C O}^{i n}\right)^{2}(1-\chi)}{\left[1+\kappa X_{C O}^{i n}(1-\chi)\right]^{2}}=0 \\
\left.\frac{1}{P e_{m}} \frac{d \chi}{d z}\right|_{z=0}=\chi(z=0),\left.\frac{d \chi}{d z}\right|_{z=1}=0
\end{gathered}
$$

For simplicity, $\kappa$ is assumed constant versus temperature. Using typical kinetic parameters for $\mathrm{CO}$ oxidation $\left(E_{a}=100 \mathrm{~kJ} / \mathrm{mol}, D a_{0}^{L H}=10^{10}, \kappa=2000, X_{C O}^{i n}=0.02\right)$, the bifurcation diagrams are calculated for different $P e_{m}$ as shown in Fig. 4. Reactors with small $P e_{m}$ have a lower ignition temperature, multiple steady-states and hence hysteresis behavior, while the full-scale reactor (high $P e_{m}$, close to PFR) does not exhibit hysteresis.

Hysteresis region caused by nonlinear kinetics is usually narrow since the nonlinearity is polynomial. It appears when $P e_{m}$ is small and $\kappa X_{C O}^{i n}$ is large. Shown in Fig. 5, are hysteresis loci for isothermal reactors with different $\kappa$ projected on the $\left(X^{i n}, P e_{m}\right)$ plane. For more "sticky" (or 


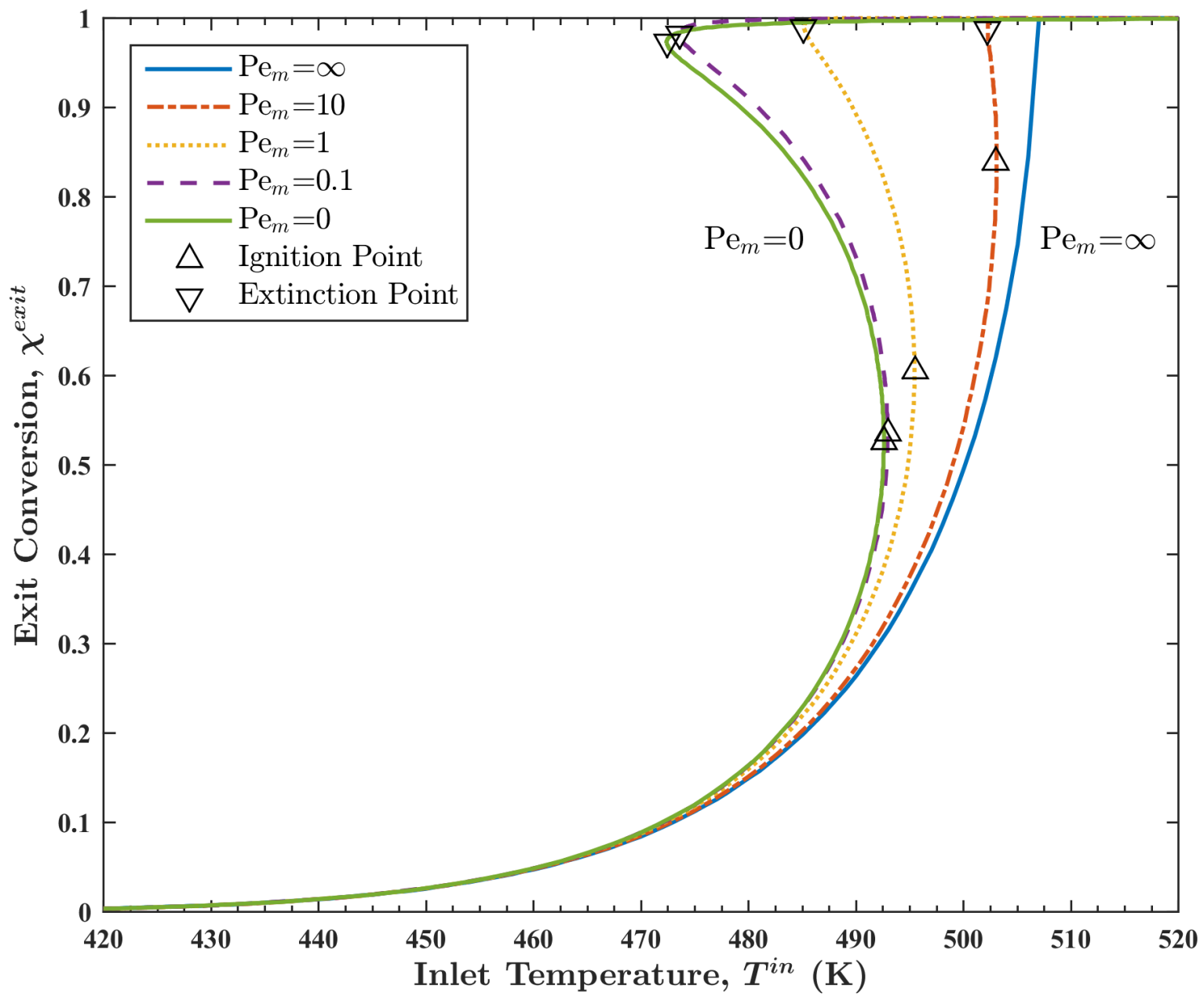

Figure 4: Bifurcation diagrams of steady-state exit conversion versus inlet temperature for isothermal reactors with Langmuir-Hinshelwood kinetics and different axial mass Péclet numbers $\left(P e_{m}\right)$. $\left(E_{a}=\right.$ $\left.100 \mathrm{~kJ} / \mathrm{mol}, D a_{0}^{L H}=10^{10}, \kappa=2000, X_{C O}^{i n}=0.02\right)$ 


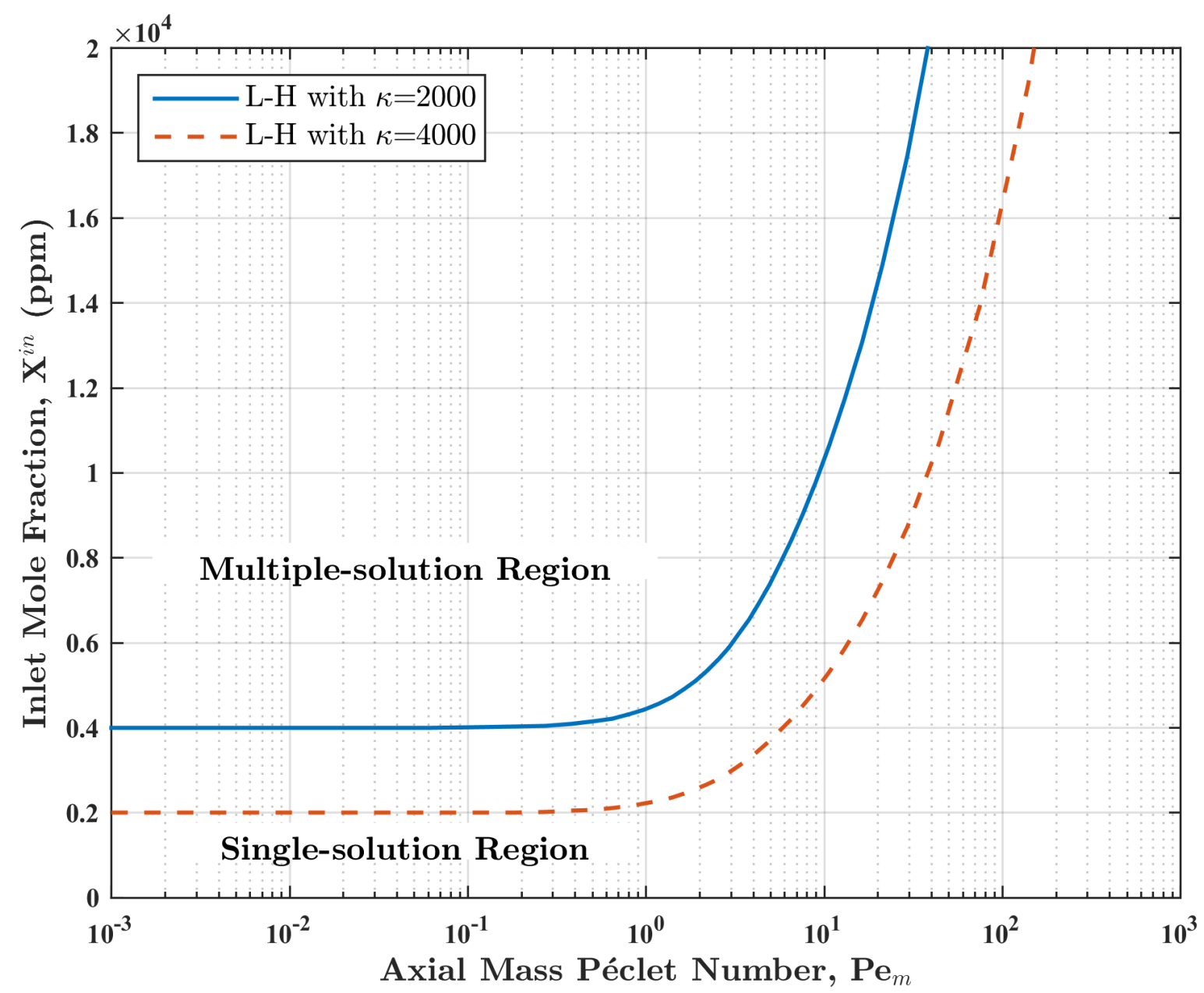

Figure 5: Hysteresis loci of isothermal reactors with Langmuir-Hinshelwood kinetics and different adsorption equilibrium constants $(\kappa)$ in the $\left(X^{i n}, P e_{m}\right)$ plane. $\left(E_{a}=100 \mathrm{~kJ} / \mathrm{mol}, D a_{0}^{L H}=10^{10}\right)$

strongly absorbed) molecules (e.g. propylene), $\kappa$ becomes larger and it is more difficult to eliminate multiplicity. When $P e_{m}$ is large $(>100)$, the inlet concentration required for multiplicity exceeds practical range and mass dispersion causes only quantitative errors. [Remark: Hysteresis locus is the set of parameter values at which ignition and extinction points appear or disappear in the bifurcation diagram of a state variable (e.g. exit conversion) versus a bifurcation parameter (e.g. inlet temperature).]

\subsection{Cycling performance}

As stated in 2.3.2, if the steady-state performances are similar in reactors of different scales, the transient performances will also be similar. Here, through simulating the cycling operations of a isothermal reactor, we illustrate that when steady-state performances of lab- and full-scale reactors 
are different, their transient performances will also differ. For a first-order reaction, the transient model is linear. The cycle averaged conversion will be almost the same as the steady-state conversion (Fig. 3) unless the cycling is very fast (cycle time is much smaller than the residence time of the reactor). For nonlinear kinetics shown, the cycling performance is analyzed by solving the transient model, (for the transient model $D a_{0}^{L H}$ is defined with respect to the average inlet mole fraction $\bar{X}_{C O}^{i n}$ )

$$
\begin{gathered}
(1+\epsilon) \frac{\partial \chi}{\partial \widehat{t}}=\frac{1}{P e_{m}} \frac{\partial^{2} \chi}{\partial z^{2}}-\frac{\partial \chi}{\partial z}+D a_{0}^{L H} \exp \left(-\frac{E_{a}}{R T^{i n}}\right) \frac{\left(1+\kappa \bar{X}_{C O}^{i n}\right)^{2}(1-\chi)}{\left[1+\kappa \bar{X}_{C O}^{i n}(1-\chi)\right]^{2}} \\
\frac{1}{P e_{m}} \frac{\partial \chi}{\partial z}-\chi=\varepsilon_{C} \sin (2 \pi \widehat{\omega} \hat{t}) \text { at } z=0, \\
\frac{\partial \chi}{\partial z}=0 \text { at } z=1 .
\end{gathered}
$$

For the simulations, we take $\epsilon=0.1, E_{a}=100 \mathrm{~kJ} / \mathrm{mol}$ and $D a_{0}=10^{10}$. As shown in Section 3.2, this model can exhibit ignition/extinction behavior with fixed $X_{C O}^{i n}$ and varying $T^{i n}$. Similarly, the model can also show ignition/extinction when $T^{i n}$ is fixed and $X_{C O}^{i n}$ is varying, which will significantly affect the cycling performances of the reactor. For example, at $T^{i n}=470 \mathrm{~K}$, bifurcation diagrams of $\chi^{\text {exit }}$ versus $X_{C O}^{i n}$ are calculated for different $P e_{m}$ as shown in Fig. 6. To better illustrate cycling performances, in the transient simulations $\varepsilon_{C}$ is chosen to be $100 \%$ so that all possible ignition/extinction behaviors are included in the calculation.

The dimensionless frequency $\widehat{\omega}$ can be interpreted as how many times the inlet concentration will cycle during the space time. For a typical after-treatment reactor, $\widehat{\omega}$ is usually smaller than 0.1 (for a $100 \mathrm{~ms}$ space time, this results in $1 \mathrm{~Hz}$ cycling). We conduct transient simulations for $\widehat{\omega}=0.01$ and 0.1 till the model reaches periodic steady-state, and plot the cycle averaged exit conversion versus inlet temperature in Fig. 7. Comparing to the steady-state light-off curve $(\widehat{\omega}=0)$, the impact of mass dispersion (the difference between lab- and full-scale reactor) is more significant in the cycling cases. Different cycling performances between lab- and full-scale reactor is also observed experimentally by Prikhodko et al. [17], who referred to this as axial length effects when they compared the performances of a 2.54-cm-long (or lab-scale) LNT catalyst and a 7.64-cm-long (or full-scale) one. 


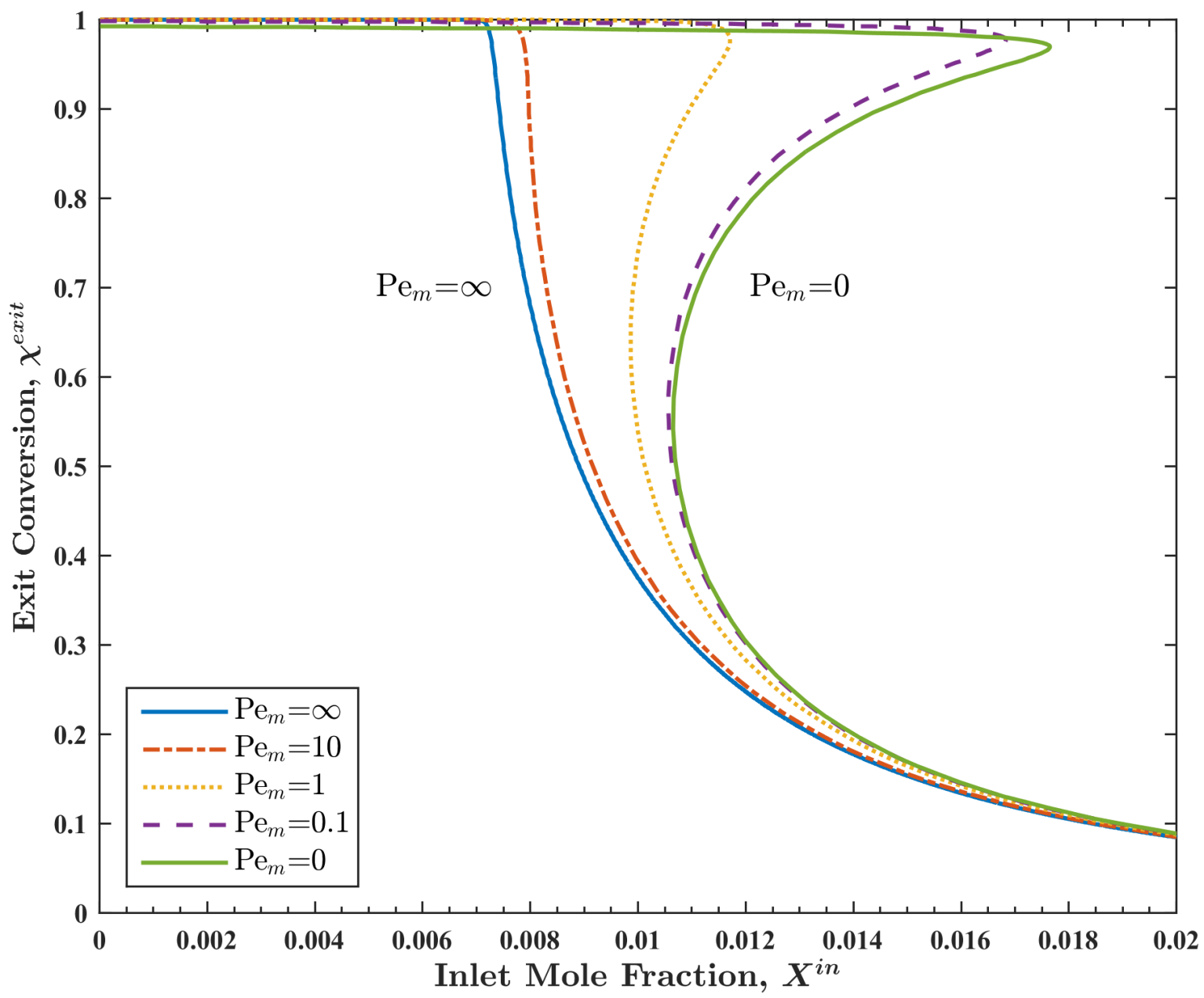

Figure 6: Bifurcation diagrams of steady-state exit conversion versus inlet mole fraction for isothermal reactors with Langmuir-Hinshelwood kinetics and different axial mass Péclet numbers $\left(P e_{m}\right) .\left(E_{a}=\right.$ $\left.100 \mathrm{~kJ} / \mathrm{mol}, D a_{0}^{L H}=10^{10}, \kappa=2000, T^{i n}=470 \mathrm{~K}\right)$ 


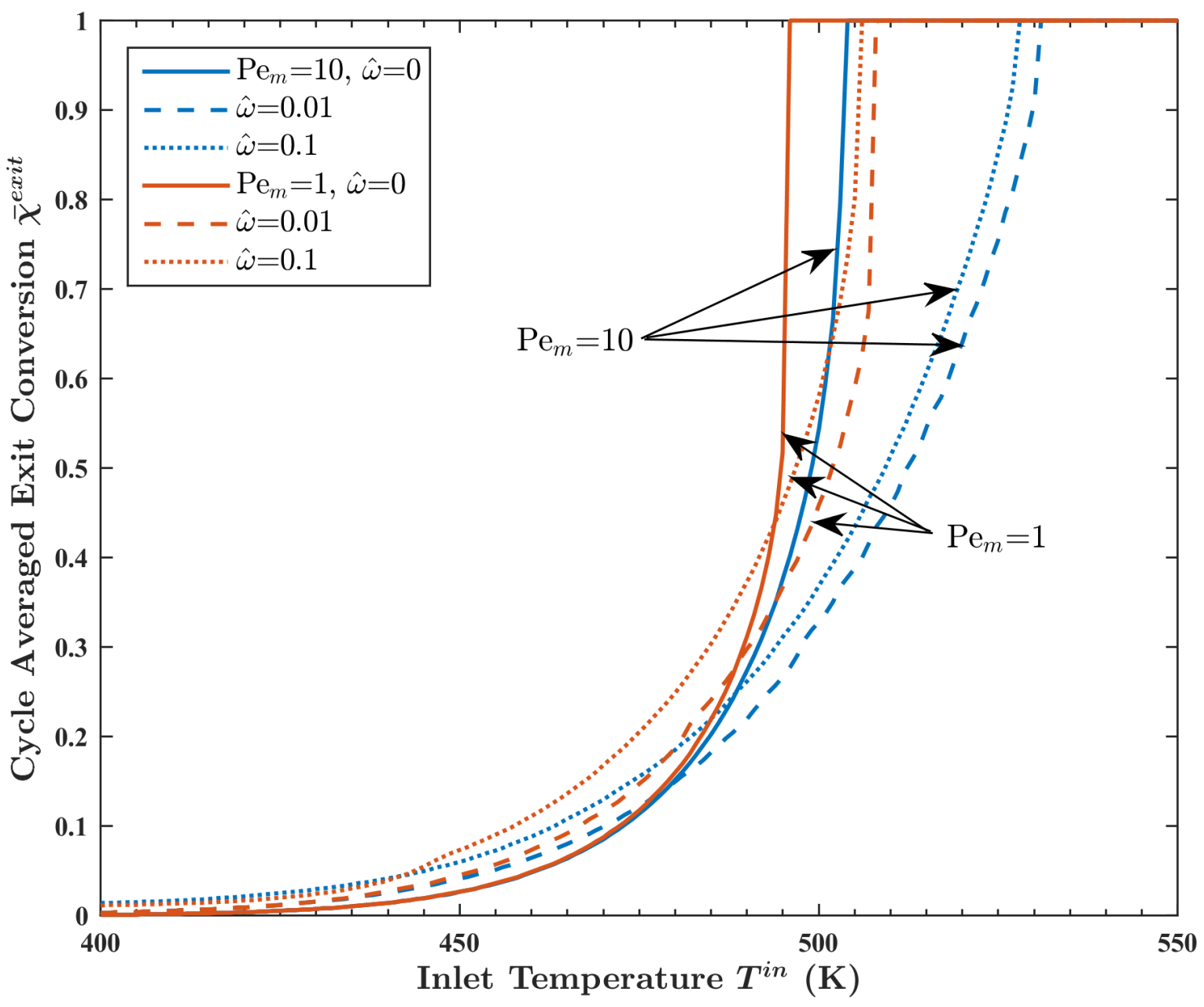

Figure 7: Light-off curves of cycle averaged exit conversion versus inlet temperature for isothermal reactors with Langmuir-Hinshelwood kinetics. $\left(E_{a}=100 \mathrm{~kJ} / \mathrm{mol}, D a_{0}^{L H}=10^{10}, \kappa=2000, \epsilon=0.1\right.$, $\left.\bar{X}_{C O}^{i n}=0.02, \varepsilon_{C}=1\right)$ 


\subsection{Estimation of kinetic parameters from light-off curves}

In after-treatment reactors, $P e_{m}$ is usually of order $10^{3}$ in full-scale reactors and is usually larger than 10 in lab-scale reactors (assuming a scaling factor of 10). Thus, in most cases mass dispersion has minor impact and the reactor behaves like a PFR. However, when the lab-scale reactor is too short (leading to small $P e_{m}$ ), axial mass dispersion is noticeably influencing the reactor behaviors. In this case, using a reactor model without axial dispersion (e.g. PFR) to estimate kinetic parameters from lab data, can result in significant quantitative errors. For linear kinetics, if we use a PFR model to fit data generated from a reactor with $P e_{m}=10$ by equalizing the values and the slopes of steady-state conversions at the inflection temperature (where the exit conversion is $1-1 / e \approx 0.632$ ), the estimated activation energy $\widetilde{E_{a}} \approx 0.923 E_{a}$, resulting in $\sim 8 \%$ error. Shown in Fig. $8 \mathrm{a}$ is how this estimation error changes with $P e_{m}$ of lab-scale reactors. In Fig. 8b, we can see that although the fitting error is small, the estimated activation energy $\widetilde{E_{a}}$ can have significant error (for the case in the figure $\sim 25 \%$ ).

When estimating kinetic parameters for nonlinear kinetics, the experiments should be designed such that both the rate constants and the adsorption constants can be estimated. The rate constants (or pre-exponential factor $A$ and activation energy $E_{a}$ ) can be obtained by using low inlet concentrations of the limiting reactant such that $K_{a d s} C^{i n} \ll 1$. In this way, the nonlinear effects are negligible and same method used in linear kinetics can be applied. After estimating $A$ and $E_{a}$, the adsorption constant $K_{a d s}$ can be estimated by using higher inlet concentration of the limiting reactant. When high concentration is used, the nonlinear effects are more pronounced and the reactor may exhibit hysteresis. In such case, both light-off (ignition) and light-out (extinction) curves should be used in the estimation. The necessity of both curves has also been emphasized in the recent work by Etheridge et al. [18]. This process has two further complications: (i) Without estimating $K_{a d s}$, one

cannot select $C^{\text {in }}$ to guarantee $K_{a d s} C^{i n} \ll 1$ and estimate $A$ and $E_{a}$ assuming a first-order reaction. Without good estimations of $A$ and $E_{a}$, accurate $K_{a d s}$ estimation cannot be obtained. Thus, the estimations of the rate constants and the adsorption constants should be done iteratively. (ii) For the negative-order effects to be significant, high inlet concentrations are used. This leads to higher adiabatic temperature rise and the isothermal assumption may not be valid. In this case, the hysteresis is caused by the combined effect of nonlinear kinetics and non-isothermal effects which is discussed 
(a)

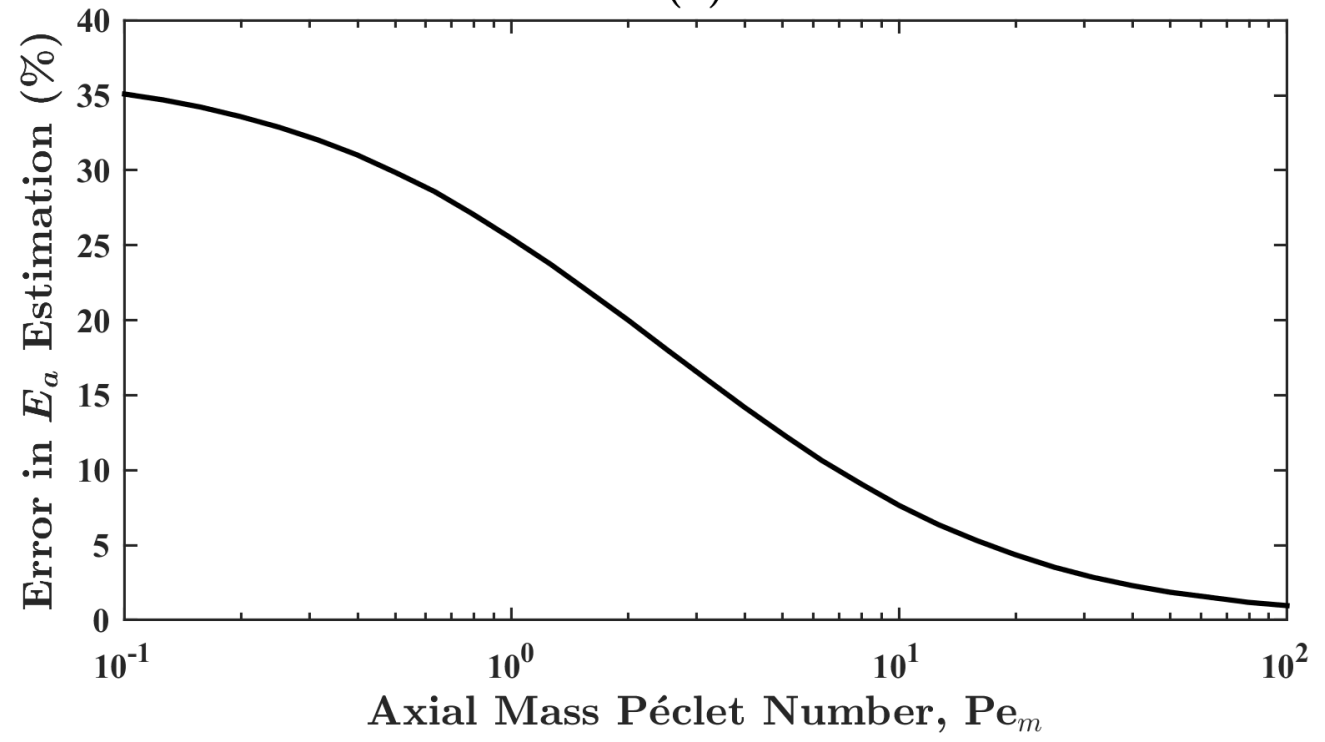

(b)

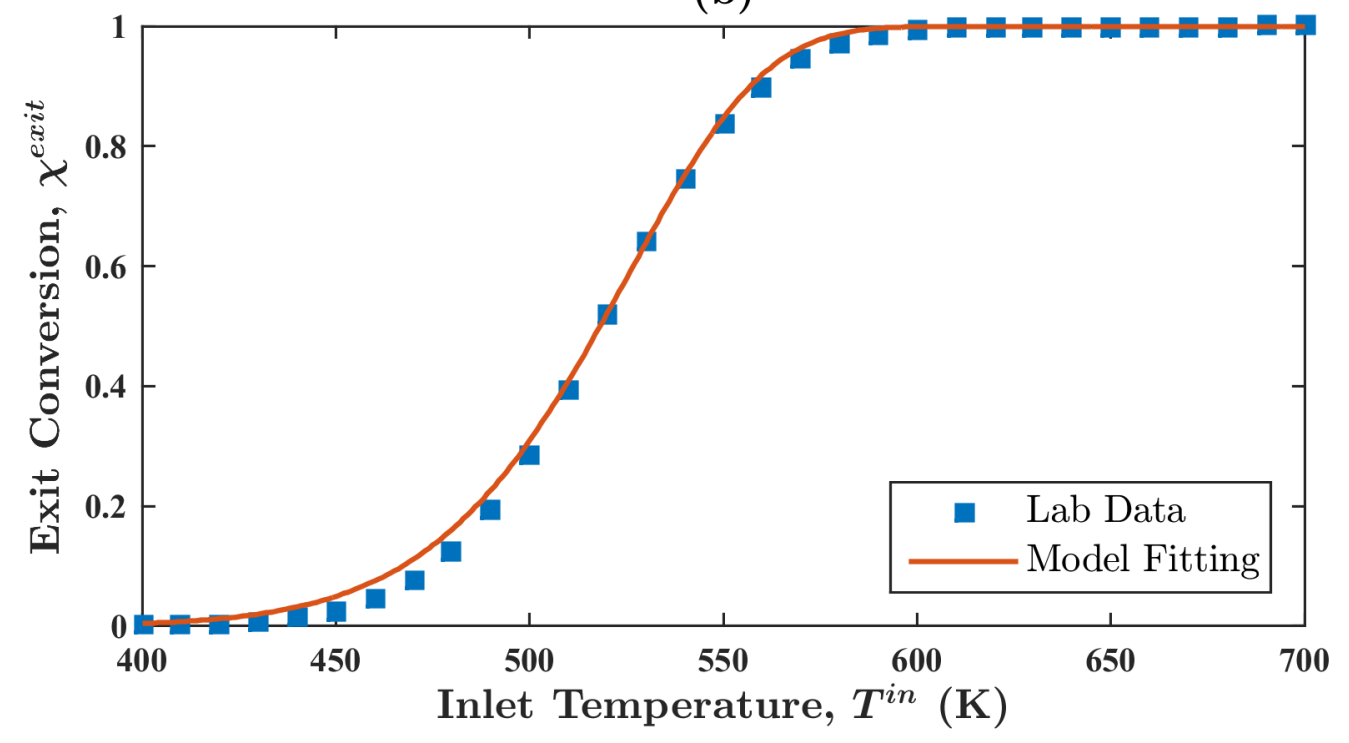

Figure 8: (a) Percentage error in the estimated activation energy by assuming a PFR model and fitting data from reactors with first-order kinetics and different $P e_{m}$. (b) PFR model fitting data generated from a reactor with $P e_{m}=1 .\left(E_{a}=100 \mathrm{~kJ} / \mathrm{mol}, D a_{0}=10^{10}\right)$ 
in Section 4.2 .

\section{Impact of heat conduction and adiabatic temperature rise}

Unlike lab-scale reactors, which are placed in a furnace whose temperature is controlled, full-scale reactors are usually operated almost adiabatically. When the adiabatic temperature rise $\Delta T_{a d}($ or $\beta$ ) is not negligible, there will be significant thermal gradients and hence an axial temperature profile in the reactor. For small $\Delta T_{a d}$, the shape of the steady-state temperature profile is determined by heat conduction. Shown in Fig. 9 are steady-state temperature profiles of adiabatic reactors with linear kinetics and different axial heat Péclet numbers $\left(P e_{h}\right)$. Larger $P e_{h}$ leads to sharper axial temperature profile. When $P e_{h}$ is large, the width of the temperature front in the non-reactive zone can be approximated as [19]

$$
\Delta z=2 \sqrt{\frac{2}{P e_{h}}} .
$$

When $\Delta T_{a d}$ (or $\beta$ ) is large, the temperature profile is affected by both heat conduction $\left(P e_{h}\right)$ and heat generation $(\beta)$. Because of the strong nonlinearity, reactors with finite $P e_{h}$ can have multiple steady-states. In order to study bifurcation behaviors, the steady-state forms of the simplified models (boundary value problems) are solved using the shooting method with sensitivity functions [20].

\subsection{Linear kinetics}

Shown in Fig. 10, are bifurcation diagrams of steady-state conversions of adiabatic reactors with different $P e_{h}$, assuming linear kinetics and $P e_{m} / P e_{h}=10$ (typical for ceramic monoliths). The $\Delta T_{a d}$ is chosen to be $200 \mathrm{~K}$, which corresponds to the oxidation of $\sim 2 \% C O$ or $\sim 3,000 \mathrm{ppm} C_{3} H_{6}$. Reactors with lower $P e_{h}$ will exhibit hysteresis and a lower ignition temperature. Thus, unless $P e_{h}$ in both lab- and full-scale reactors are very large, their behaviors will be qualitatively different.

Typically in full-scale ceramic monolith reactors, $P e_{h} \sim 100$ leading to a PFR-like behavior, while $P e_{h}$ in lab-scale reactors are 10 to 100 times smaller leading to hysteresis behaviors. In fullscale metallic monolith reactors where $P e_{h} \sim 10$, there can be hysteresis in both lab- and full-scale 


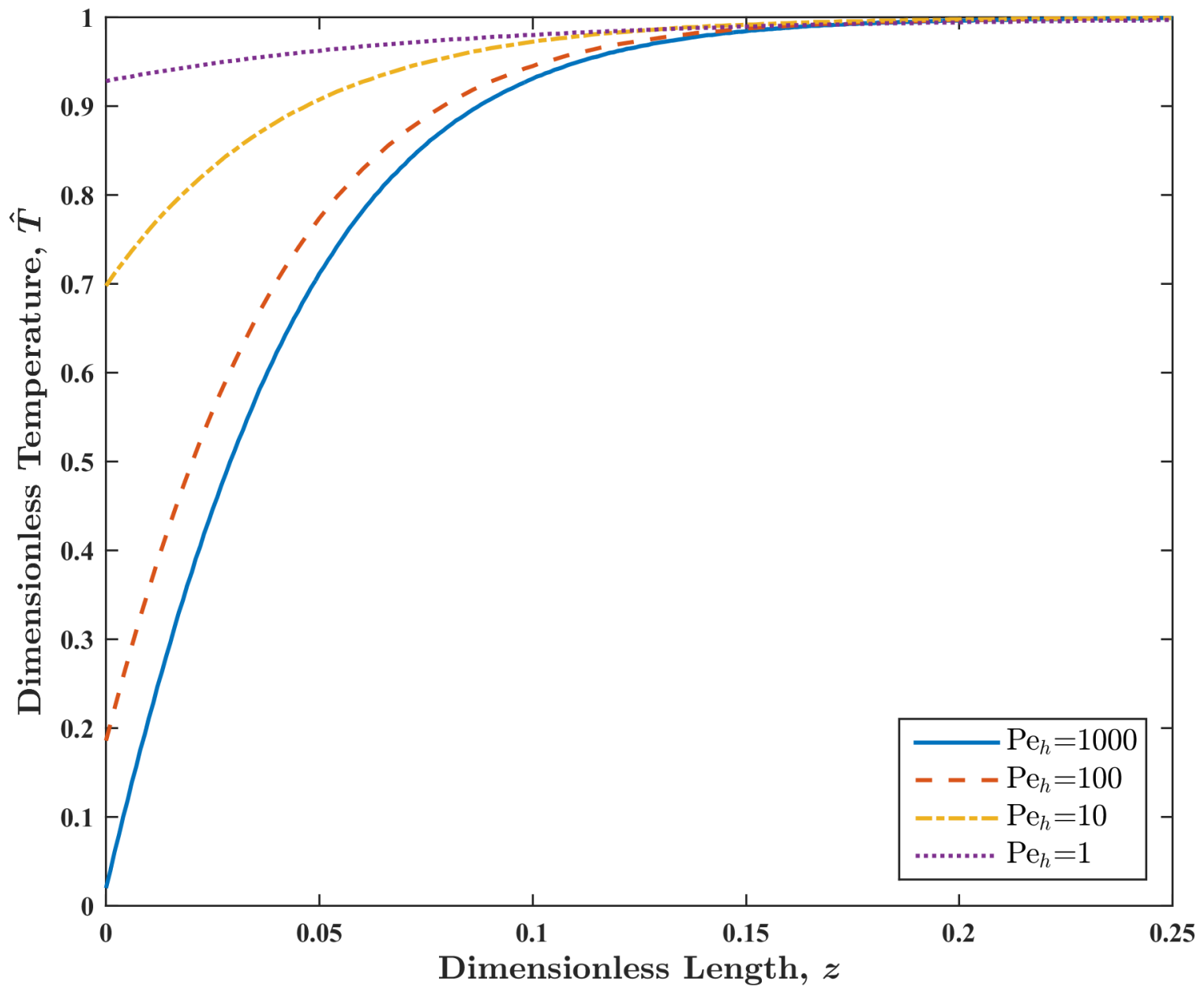

Figure 9: Steady-state axial temperature profiles of adiabatic reactors with first-order kinetics and different axial heat Péclet numbers $\left(P e_{h}\right) .\left(E_{a}=100 \mathrm{~kJ} / \mathrm{mol}, D a_{0}=10^{10}, \Delta T_{a d}=12 \mathrm{~K}, T^{i n}=\right.$ $\left.600 \mathrm{~K}, P e_{m} / P e_{h}=10\right)$ 


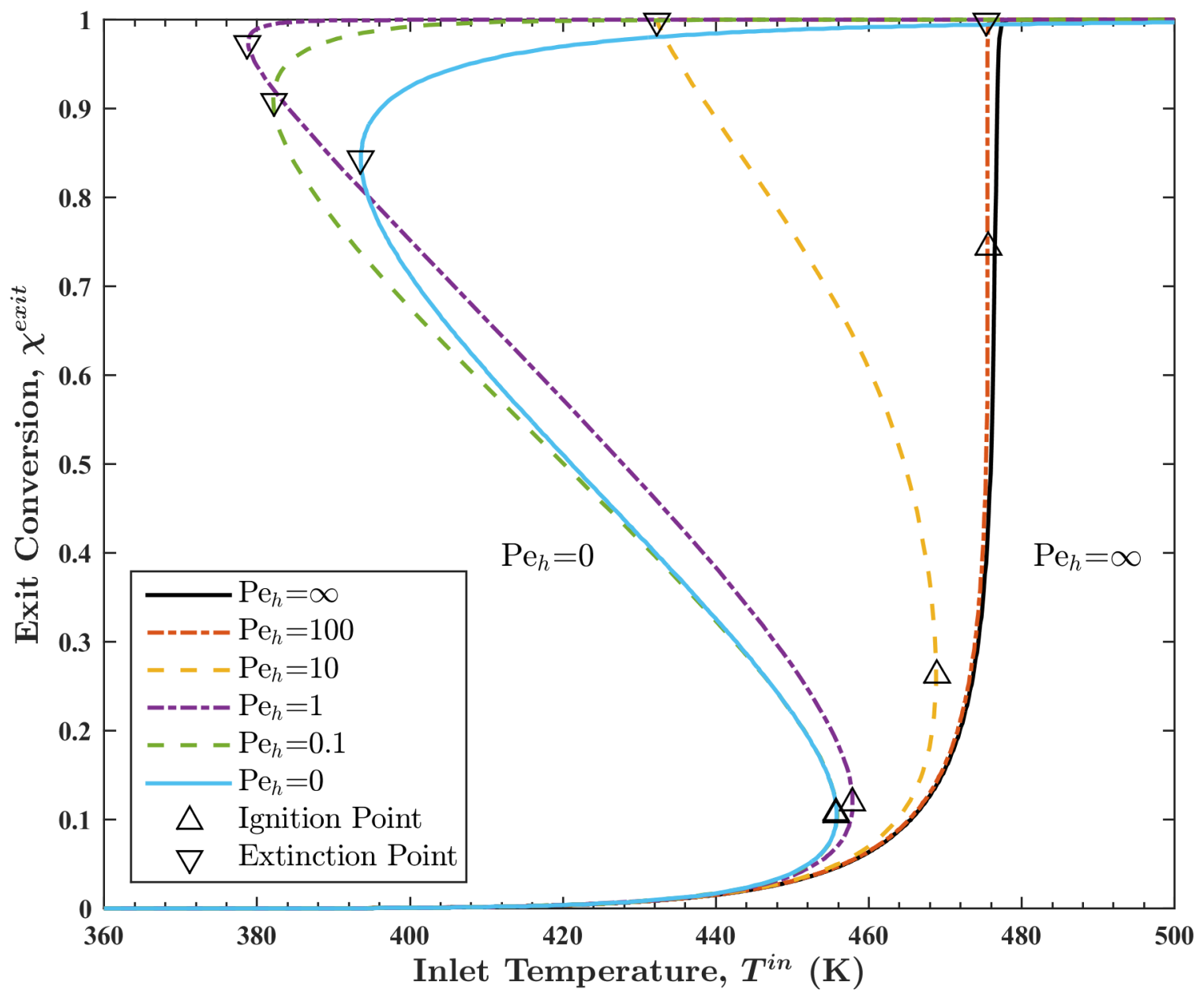

Figure 10: Bifurcation diagrams of steady-state exit conversion versus inlet temperature in adiabatic reactors with first-order kinetics and different axial heat Péclet numbers $\left(P e_{h}\right) .\left(E_{a}=100 \mathrm{~kJ} / \mathrm{mol}\right.$, $\left.D a_{0}=10^{10}, \Delta T_{a d}=200 \mathrm{~K}, P e_{m} / P e_{h}=10\right)$ 
reactors, but the hysteresis in lab-scale will be more pronounced. Thus, a direct scale-up based on lab-scale performance is usually not possible, because the estimated ignition temperature will be lower. Also, the ignited branch, where the steady-state conversion can stay high even when the inlet temperature drops, does not exist or shrinks in the full-scale reactors. It can be noted from Fig. 10 that when $P e_{h}$ is very small, the width of the hysteresis region does not decrease monotonically with increase in $P e_{h}$. The reason for this may be that when $P e_{h}$ is sufficiently small (suggesting that temperature profile is close to uniform), $P e_{m}=10 P e_{h}$ is still large enough to create a relatively sharp concentration profile, as illustrated in Fig. 11, hence for a certain $P e_{h}$ range the model is approaching the lumped thermal reactor asymptote which has a larger hysteresis region [21].

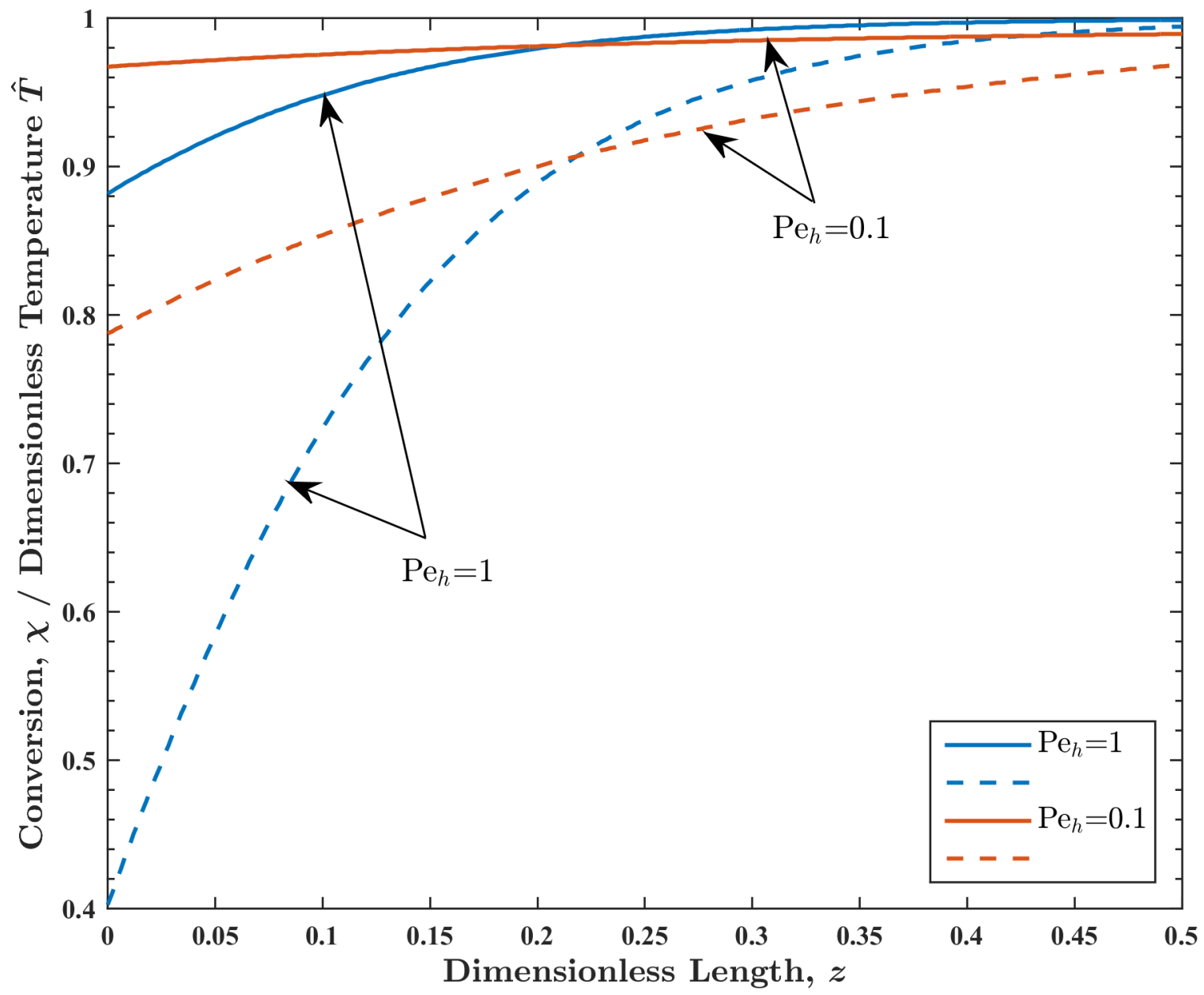

Figure 11: Steady-state axial temperature (solid line) and conversion (dashed line) profiles of adiabatic reactors with first-order kinetics and small $P e_{h}$. $\left(E_{a}=100 \mathrm{~kJ} / \mathrm{mol}, D a_{0}=10^{10}, \Delta T_{a d}=200 \mathrm{~K}\right.$, $\left.T^{i n}=400 \mathrm{~K}, P e_{m} / P e_{h}=10\right)$

The width of the hysteresis region is also impacted by the thermal effects $\left(\Delta T_{a d}\right)$, since the more heat is generated, the lower inlet temperature is needed to maintain high steady-state conversion. To 
illustrate this effect, we calculated the bifurcation sets (ignition/extinction loci) of adiabatic reactors at different $P e_{h}$ as shown in Fig. 12. After $\Delta T_{a d}$ exceeds a certain value (hysteresis point), the width of the hysteresis region increases monotonically with $\Delta T_{a d}$. From Fig. 12, we can see that the ignition locus is almost vertical suggesting that the ignition temperatures are not sensitive to the thermal effects. Thus, the widening of the hysteresis region is mainly in the form of lower extinction temperatures.

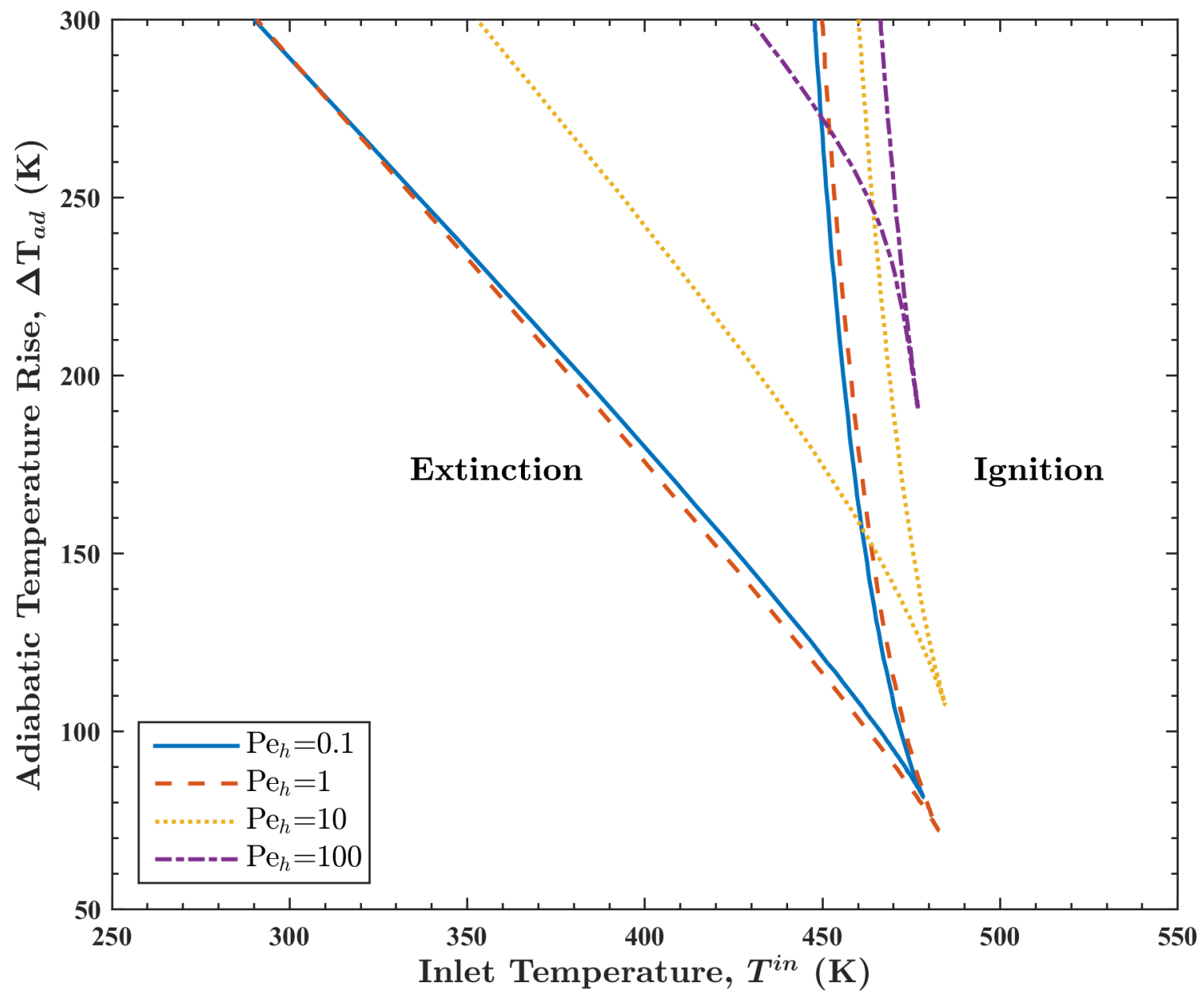

Figure 12: Ignition/extinction loci of adiabatic reactors with first-order kinetics and different axial heat Péclet numbers $\left(P e_{h}\right) .\left(E_{a}=100 \mathrm{~kJ} / \mathrm{mol}, D a_{0}=10^{10}, P e_{m} / P e_{h}=10\right)$

The hysteresis points are of special interests, since they indicate the boundary of single- and multiple-solution regions and determine whether the differences between lab- and full-scale reactors are qualitative. Shown in Fig. 13, are hysteresis loci of adiabatic reactors with different $P e_{m} / P e_{h}$ ratios projected on the $\left(\Delta T_{a d}, P e_{h}\right)$ plane. For larger $P e_{h}$, the adiabatic temperature rise required for hysteresis increases exponentially with $P e_{h}[22]$. The hysteresis loci of reactor with $P e_{m} / P e_{h}=1$ 
(more realistic for packed bed reactors) and $P e_{m} / P e_{h}=\infty$ (mass dispersion is neglected) divide the plane into three regions. The region (painted in blue) above the hysteresis locus with $P e_{m} / P e_{h}=1$ is the multiple-solution region; the region (painted in red) below the hysteresis locus with $P e_{m} / P e_{h}=$ $\infty$ is the single-solution region. The region in between these two lines is the transitional region where the material of the monolith $\left(P e_{m} / P e_{h}\right.$ ratio) determines whether the reactor exhibits hysteresis. For larger $P e_{h}$, the hysteresis locus with $P e_{m} / P e_{h}=\infty$ is a good approximation for both ceramic and metallic monolith reactors. In Fig. 13, some typical operation points are marked as stars. The blue six-pointed star indicates a full-scale ceramic reactor with $\Delta T_{a d}=200 \mathrm{~K}$. The red five-pointed star indicates a full-scale metallic reactor or a lab-scale ceramic reactor with $1 / 3$ of the full length. The green four-pointed star indicates a lab-scale metallic reactor with $1 / 3$ of the full length or a lab-scale ceramic reactor with 1/10 of the full length. For qualitative similarity, lab- and full-scale reactors should fall in the same region. Lab- and full-scale ceramic reactors often lie in different regions indicating qualitative differences. Although lab- and full-scale metallic reactors may lie in the same region, the qualitative difference is usually significant as shown in Figs. 10 and 12. The hysteresis loci are also projected on $\left(\chi^{e x i t}, P e_{h}\right)$ and $\left(T^{i n}, P e_{h}\right)$ planes as shown in Figs. 14 and 15. These loci show how steady-state exit conversion $\chi^{\text {exit }}$ and inlet temperature $T^{i n}$ at the hysteresis point vary with $P e_{h}$. As shown in Fig. 12, $T^{i n}$ at hysteresis point is a good approximation for the ignition temperature, so Fig. 15 can also be interpreted as how ignition temperature varies with $P e_{h}$.

\subsection{Nonlinear kinetics}

In Section 4.1, we assumed the reaction is first-order, thus the nonlinearity is only from the exponential temperature dependence of the reaction rate. However, the nonlinearity can also appear in the rate expressions as we have shown in Section 3.2 for the isothermal case. Here, we show how nonlinear mechanism is coupled with non-isothermal effects. For the Langmuir-Hinshelwood kinetics, we assume the reactant is $C O$, the dimensionless adsorption equilibrium constant $\kappa=2000$ and $\Delta T_{a d} \approx 10^{-2} X^{i n}$ (in ppm). As shown in Fig. 16, the combined effect of Langmuir-Hinshelwood kinetics and non-isothermal effects leads to a hysteresis region that is significantly wider than single effect cases. Taking the limit $P e_{h} \rightarrow 0$ for example, the width of hysteresis region for adiabatic op- 


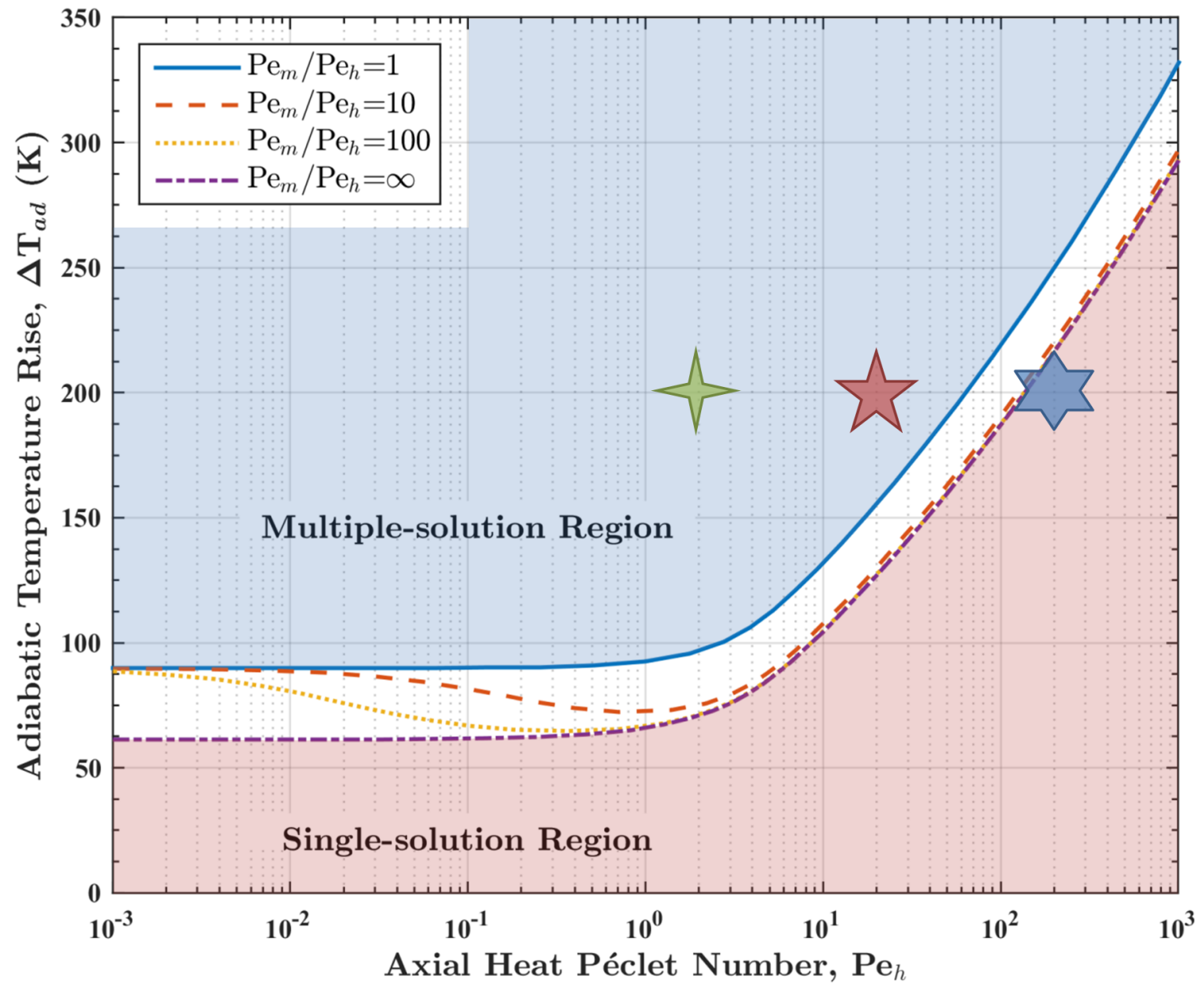

Figure 13: Hysteresis loci of adiabatic reactors with first-order kinetics and different $P e_{m} / P e_{h}$ ratios in the $\left(\Delta T_{a d}, P e_{h}\right)$ plane. $\left(E_{a}=100 \mathrm{~kJ} / \mathrm{mol}, D a_{0}=10^{10}\right)$ 


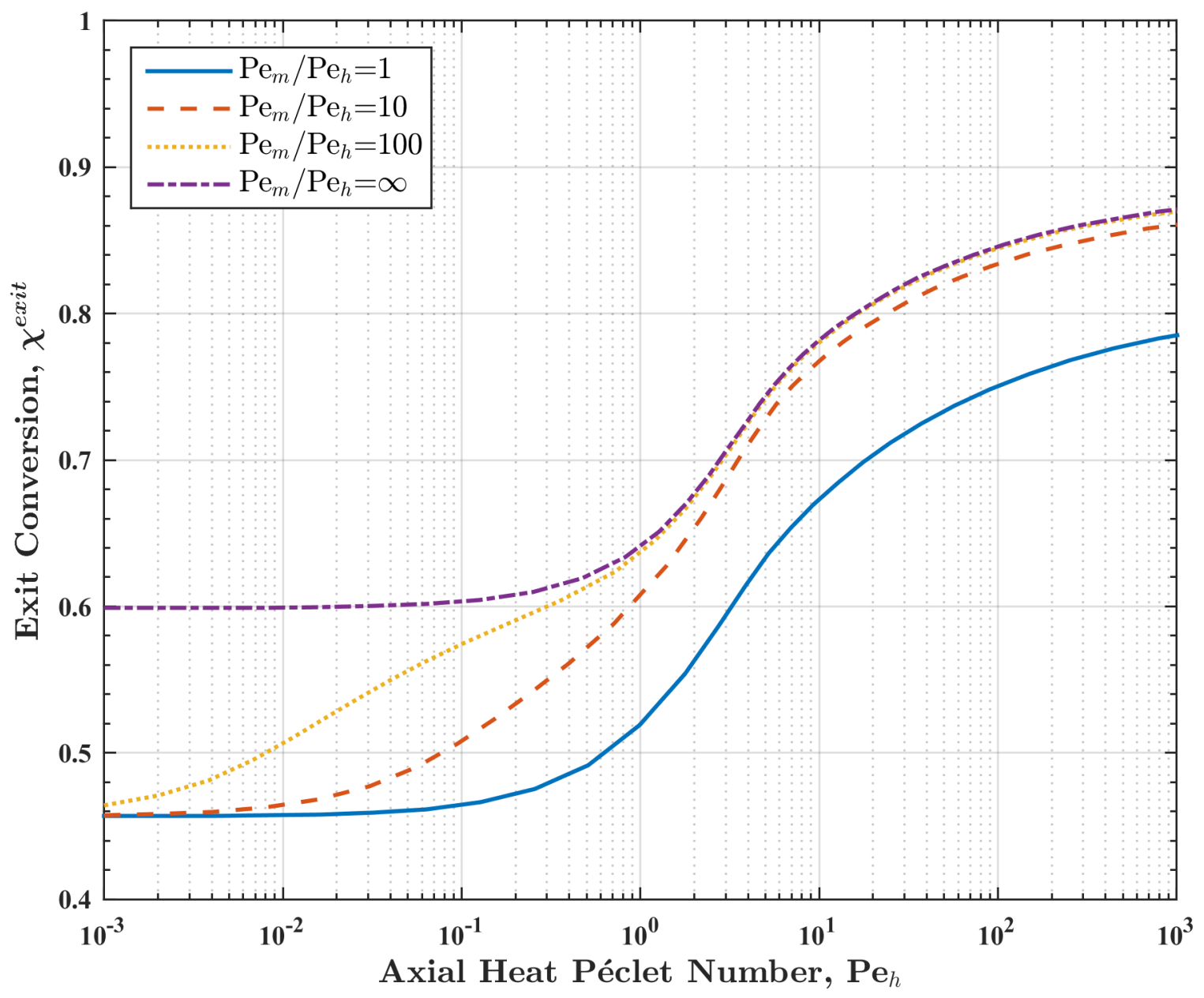

Figure 14: Hysteresis loci of adiabatic reactors with first-order kinetics and different $P e_{m} / P e_{h}$ ratios in the $\left(\chi^{\text {exit }}, P e_{h}\right)$ plane.$\left(E_{a}=100 \mathrm{~kJ} / \mathrm{mol}, D a_{0}=10^{10}\right)$ 


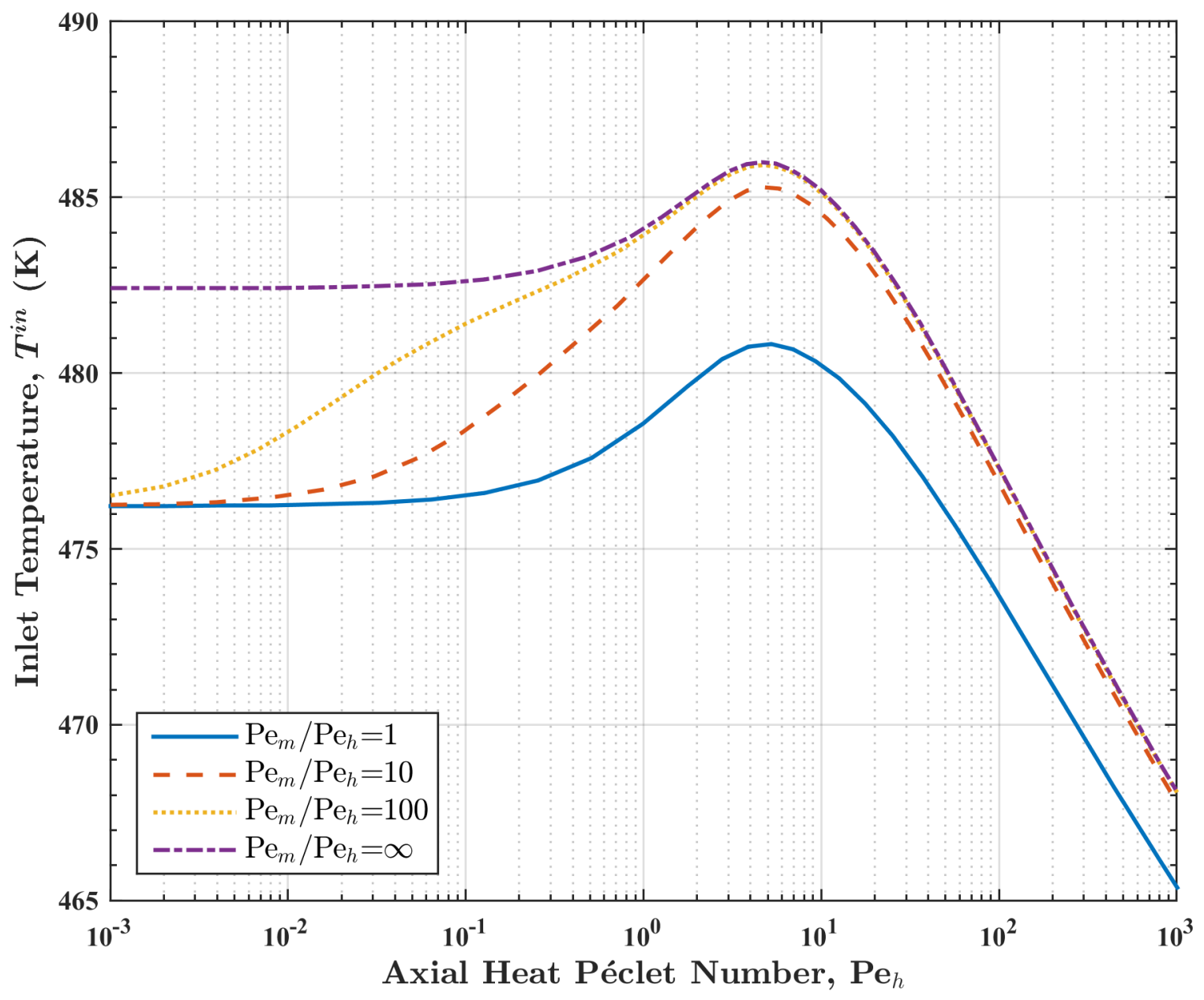

Figure 15: Hysteresis loci of adiabatic reactors with first-order kinetics and different $P e_{m} / P e_{h}$ ratios in the $\left(T^{i n}, P e_{h}\right)$ plane. $\left(E_{a}=100 \mathrm{~kJ} / \mathrm{mol}, D a_{0}=10^{10}\right)$ 


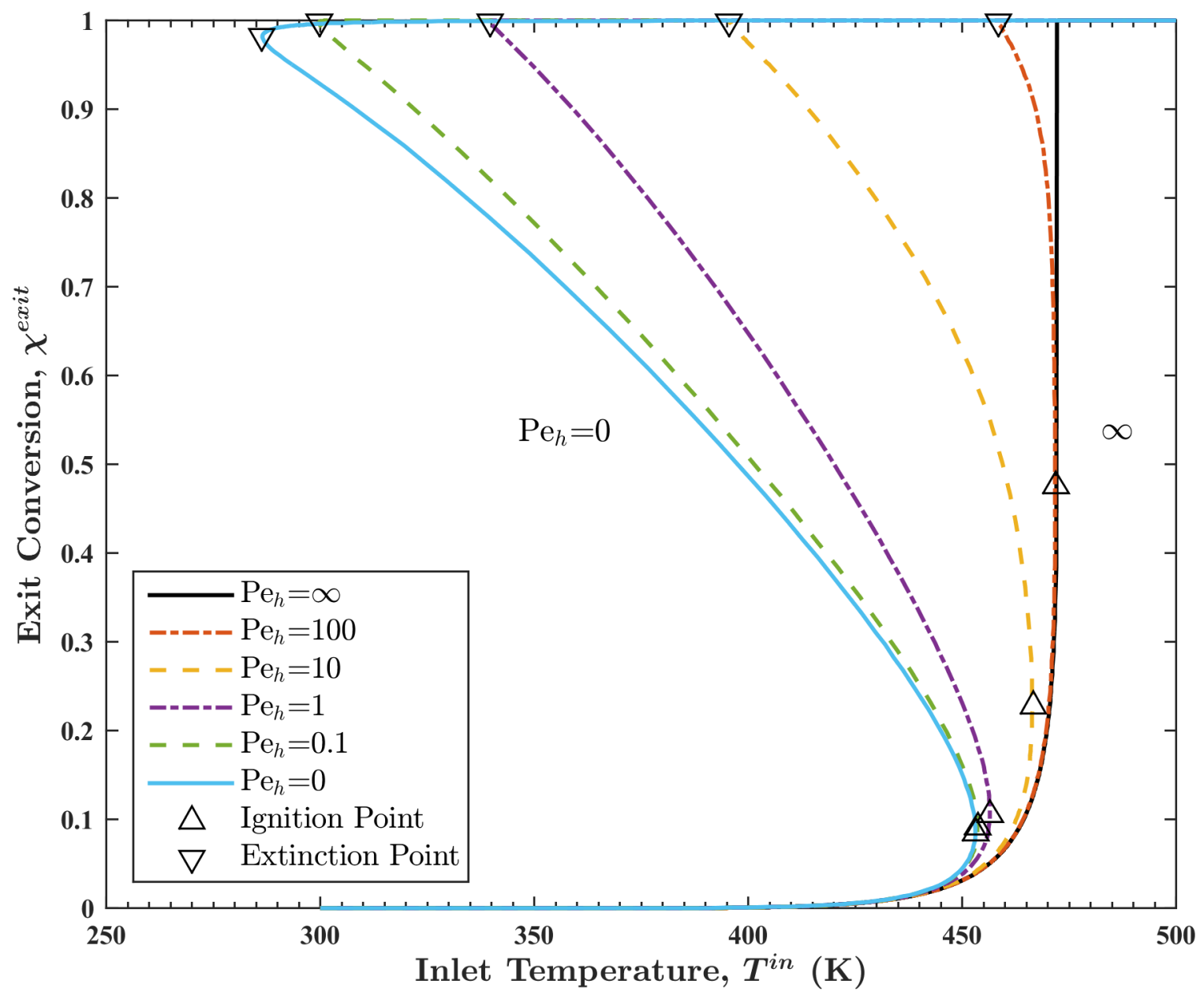

Figure 16: Bifurcation diagrams of steady-state exit conversion versus inlet temperature in adiabatic reactors with Langmuir-Hinshelwood kinetics and different axial heat Péclet numbers $\left(P e_{h}\right)$. $\left(E_{a}=\right.$ $\left.100 \mathrm{~kJ} / \mathrm{mol}, D a_{0}^{L H}=10^{10}, \kappa=2000, X_{C O}^{i n}=0.02, P e_{m} / P e_{h}=10\right)$

eration with Langmuir-Hinshelwood kinetics is $167 \mathrm{~K}$ compared to $62 \mathrm{~K}$ for adiabatic operation with first-order reaction (non-isothermal effects alone) and $20 \mathrm{~K}$ for isothermal operation with LangmuirHinshelwood kinetics (nonlinear kinetics alone).

Nonlinear kinetics also affect the boundary of multiple-solution region. To illustrate this effect, we calculated the hysteresis loci and projected them on the $\left(X^{i n}, P e_{h}\right)$ plane as shown in Fig. 17. The general trends of the loci are the same. However, comparing to first-order reaction case, the nonlinear kinetics significantly enlarged the multiple-solution region. Comparing to the isothermal case, thermal effects enlarged the multiple-solution region, especially for large $P e_{h}$. A case with $\kappa=200$ is also calculated to show the transition from first-order to strong nonlinear kinetics. 


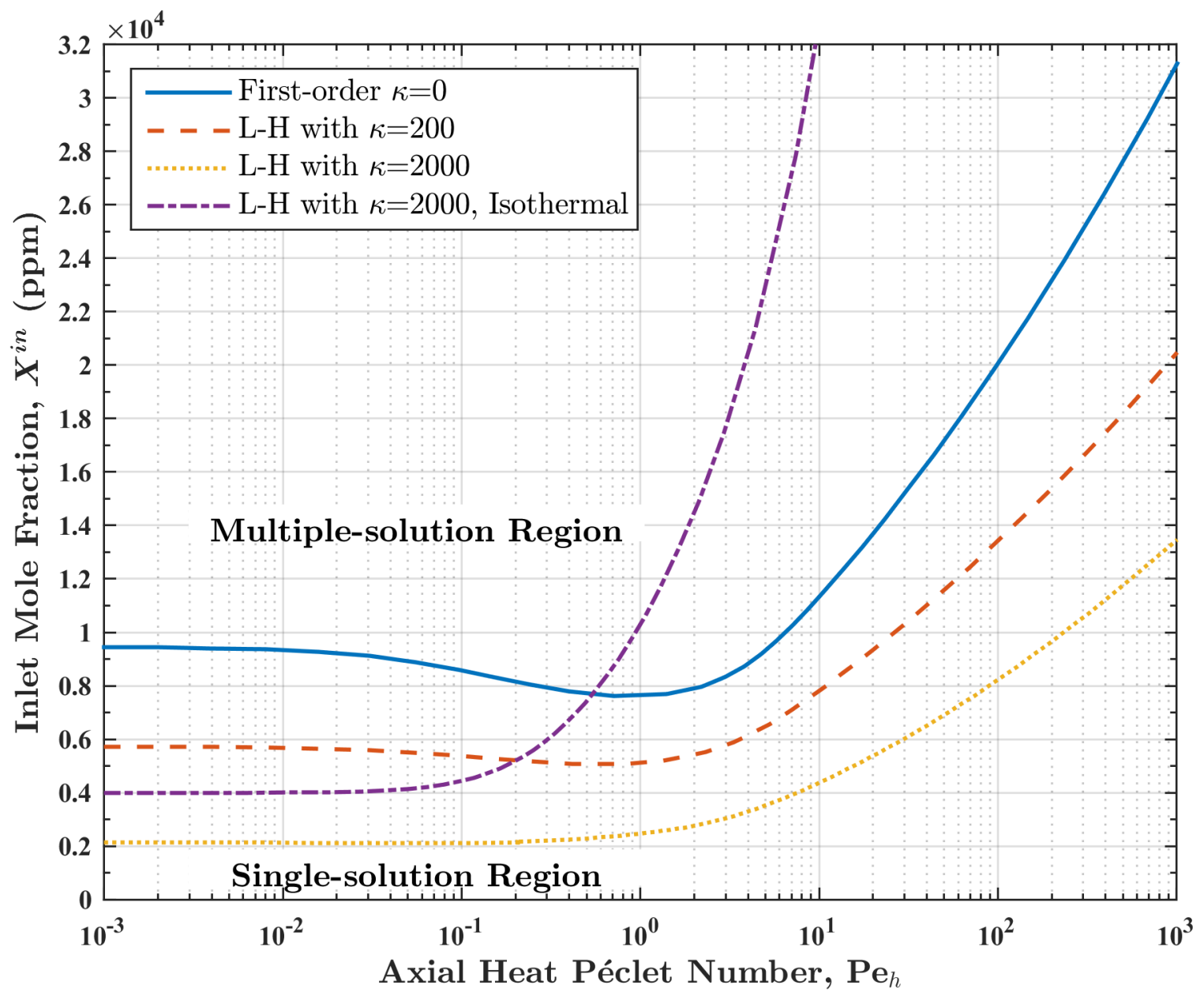

Figure 17: Hysteresis loci of adiabatic reactors with Langmuir-Hinshelwood kinetics and different adsorption equilibrium constants $(\kappa)$ on the $\left(X^{i n}, P e_{h}\right)$ plane. $\left(E_{a}=100 \mathrm{~kJ} / \mathrm{mol}, D a_{0}^{L H}=10^{10}\right.$, $\left.P e_{m} / P e_{h}=10\right)$ 


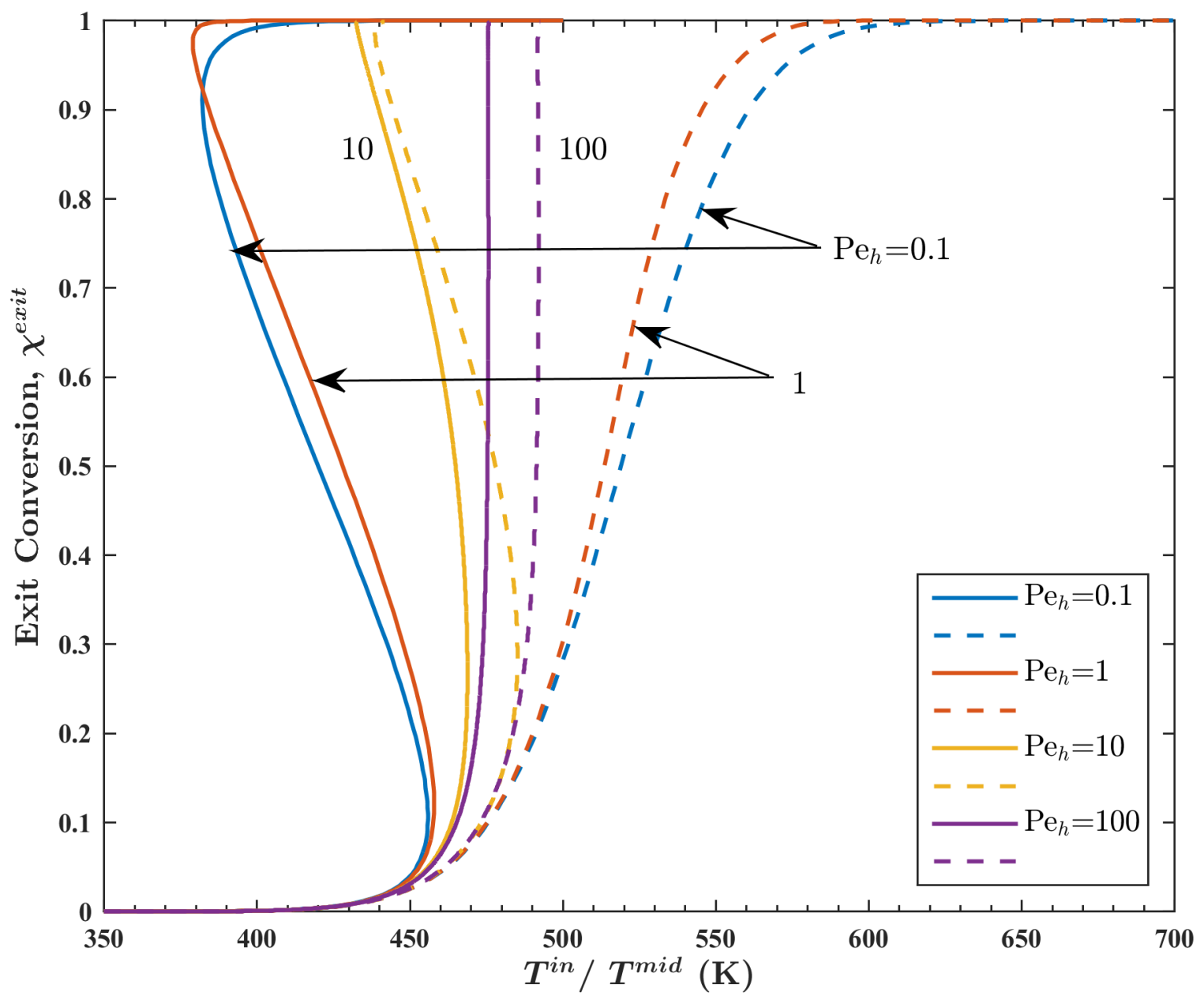

Figure 18: Bifurcation diagrams of steady-state exit conversion versus inlet temperature (solid) / mid-bed temperature (dashed) in adiabatic reactors with first-order kinetics and different axial heat Péclet numbers $\left(P e_{h}\right) .\left(E_{a}=100 \mathrm{~kJ} / \mathrm{mol}, D a_{0}=10^{10}, \Delta T_{a d}=200 \mathrm{~K}, P e_{m} / P e_{h}=10\right)$

\subsection{Plots of exit conversion versus mid-bed temperature}

Laboratory-scale experimental data are often displayed by plotting the limiting reactant exit conversion as a function of the catalyst temperature at the mid-point of the monolith. The main reason for such plots is the belief that the kinetics can be determined more accurately if the temperature profile within the monolith is uniform. We examine here the validity of this assumption, especially for cases in which $\Delta T_{a d}$ is not small.

Shown in Fig. 18, are the bifurcation diagrams of steady-state conversion versus inlet temperature (same as Fig. 10) and mid-bed temperature $\left(T^{\text {mid }}\right)$. We can see that for small $P e_{h}$ the curve of $\chi^{\text {exit }}$ versus $T^{\text {mid }}$ is single-valued and very similar to the isothermal reactor case (Fig. 3). This might lead to the belief that these plots are better for kinetic studies, since they can be treated as isothermal 
reactors at mid-bed temperatures. However, this type of plot is not a good way to present the results, because $T^{\text {mid }}$ itself is an output of the system (unlike $T^{\text {in }}$ which is an input) which is affected by inlet temperature, kinetics and adiabatic temperature rise $\left(T^{m i d}=T^{i n}+\chi^{m i d} \Delta T_{a d}\right)$. Plotting two outputs versus each other may conceal the actual system behavior. More importantly, the singlevalued plot of $\chi^{\text {exit }}$ versus $T^{\text {mid }}$ can be misleading, since part of the curve is unstable and cannot be observed experimentally. To illustrate this, we take the $P e_{h}=0.1$ curves in Fig. 18 for example and plot them again in Fig. 19 with dotted part indicating unstable steady-states. By either slowly increasing or decreasing the inlet temperature, one can find either the light-off curve (upward arrows) or light-out curve (downward arrows). Thus, although the $\chi^{\text {exit }}$ versus $T^{\text {mid }}$ curve is single-valued in the bifurcation diagram, it still has hysteresis (as long as the corresponding $\chi^{\text {exit }}$ versus $T^{\text {in }}$ curve is multi-valued). Thus, we believe that $\chi^{\text {exit }}$ versus $T^{\text {mid }}$ plots provide no significant advantage over $\chi^{\text {exit }}$ versus $T^{\text {in }}$ plots.

In Fig. 18, we can also see for the same $\chi^{\text {exit }}$, the difference between $T^{i n}$ and $T^{m i d}$ decreases as $P e_{h}$ increases. This is due to shifting of the reaction zone. To illustrate this, we plot the axial temperature profiles of reactors with different $P e_{h}$, at their extinction points (lowest possible temperature to have high conversion) in Fig. 20. For smaller $P e_{h}$, high conversion can be achieved near the inlet of the reactor and the temperature profile is near flat, thus $T^{\text {mid }}-T^{i n} \approx T^{\text {exit }}-T^{i n}=\chi^{\text {exit }} \Delta T_{a d}$. For larger $P e_{h}$, high conversion is achieved only near the end of the reactor. Thus, even when exit conversion is high, $\chi^{\text {mid }}$ is still small and the difference between $T^{i n}$ and $T^{\text {mid }}$ will also be small. After the exit conversion reaches unity, if $T^{\text {in }}$ keeps increasing, the reaction zone will shift towards the inlet and ultimately the profile will also become flat.

We note that multiple solutions disappear and the curve of $\chi^{\text {exit }}$ versus $T^{\text {in }}$ or $\chi^{\text {exit }}$ versus $T^{\text {mid }}$ are single-valued if $\Delta T_{a d}$ is below the hysteresis locus in Fig. 13. However, to achieve a small enough $\Delta T_{a d}$ the inlet reactant concentration may become so low that the measurement accuracy may be impacted. In such cases, the results presented in this work may be used to select the proper catalyst dimensions and reactant concentrations to obtain meaningful laboratory data that can be used for kinetic parameter estimation. 


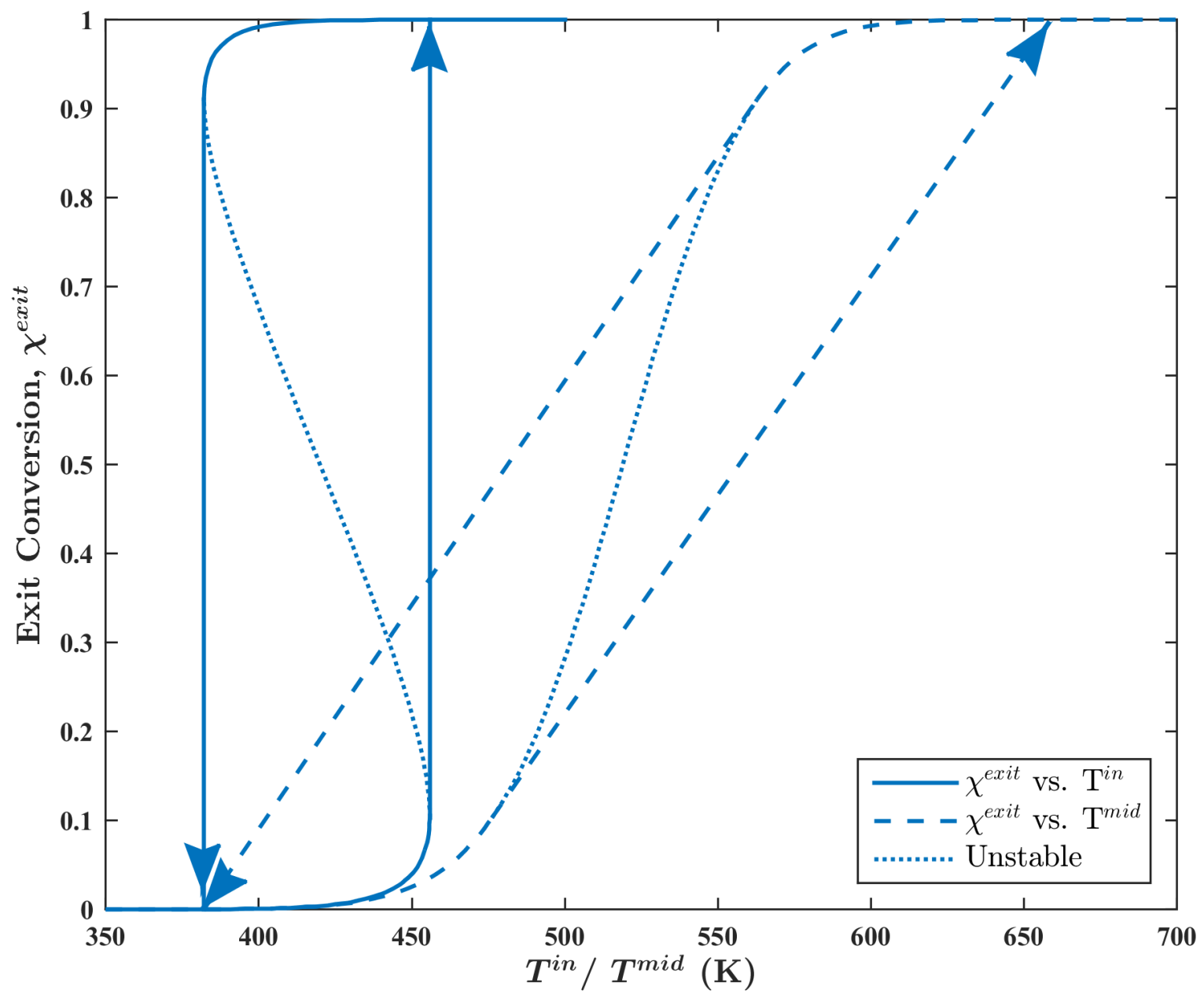

Figure 19: Bifurcation diagrams of steady-state exit conversion versus inlet temperature (solid) / mid-bed temperature (dashed) in adiabatic reactors with first-order kinetics. $\left(E_{a}=100 \mathrm{~kJ} / \mathrm{mol}\right.$, $\left.D a_{0}=10^{10}, \Delta T_{a d}=200 \mathrm{~K}, P e_{h}=0.1, P e_{m}=1\right)$ 


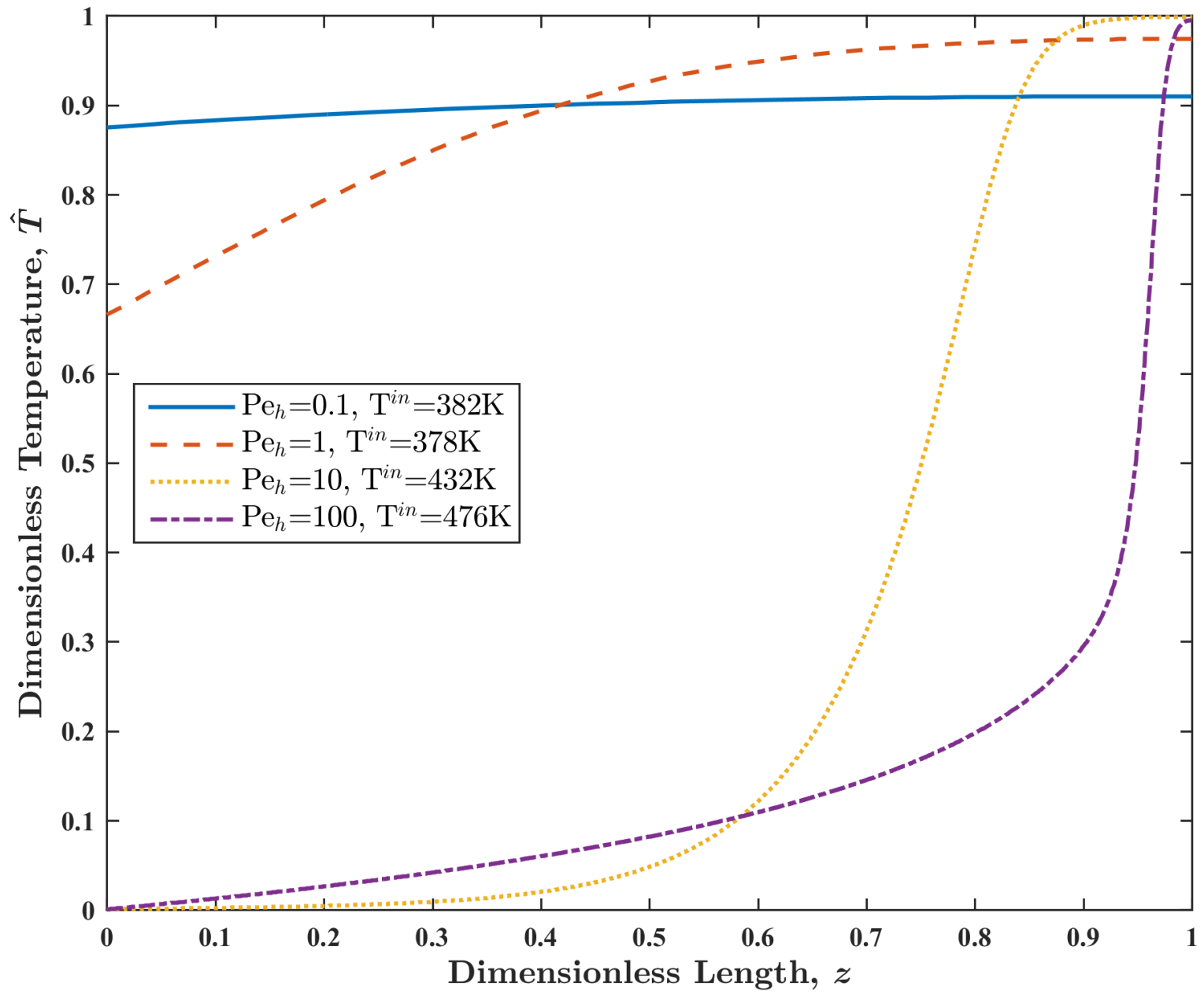

Figure 20: Steady-state axial temperature profiles of adiabatic reactors with first-order kinetics and different axial heat Péclet numbers $\left(P e_{h}\right)$ at extinction temperatures. $\left(E_{a}=100 \mathrm{~kJ} / \mathrm{mol}, D a_{0}=10^{10}\right.$, $\left.\Delta T_{a d}=200 \mathrm{~K}, P e_{m} / P e_{h}=10\right)$ 


\section{Impact of interphase gradients}

Although the analysis so far is mainly based on a one-dimensional pseudo-homogeneous reactor model (described in Section 2.3.2), the conclusions are valid for small excursions from the assumptions. Here, we examine the impact of interphase (or radial) gradients. In the pseudo-homogeneous model, the transverse Péclet numbers are either neglected $\left(P_{m}=P_{h}=0\right)$ or lumped into effective Péclet numbers. Since the typical $P_{m}$ value of an after-treatment reactor is of the order 0.01 , the assumption is usually valid. As shown in Fig. $21, P_{m}=P_{h}=0.01$ leads to negligible deviation from the pseudohomogeneous model. Even when the interphase gradients are larger $\left(P_{m}=P_{h}=0.1\right.$ which is one order of magnitude larger than typical values), the pseudo-homogeneous model can predict the ignition and extinction temperatures with only small errors.

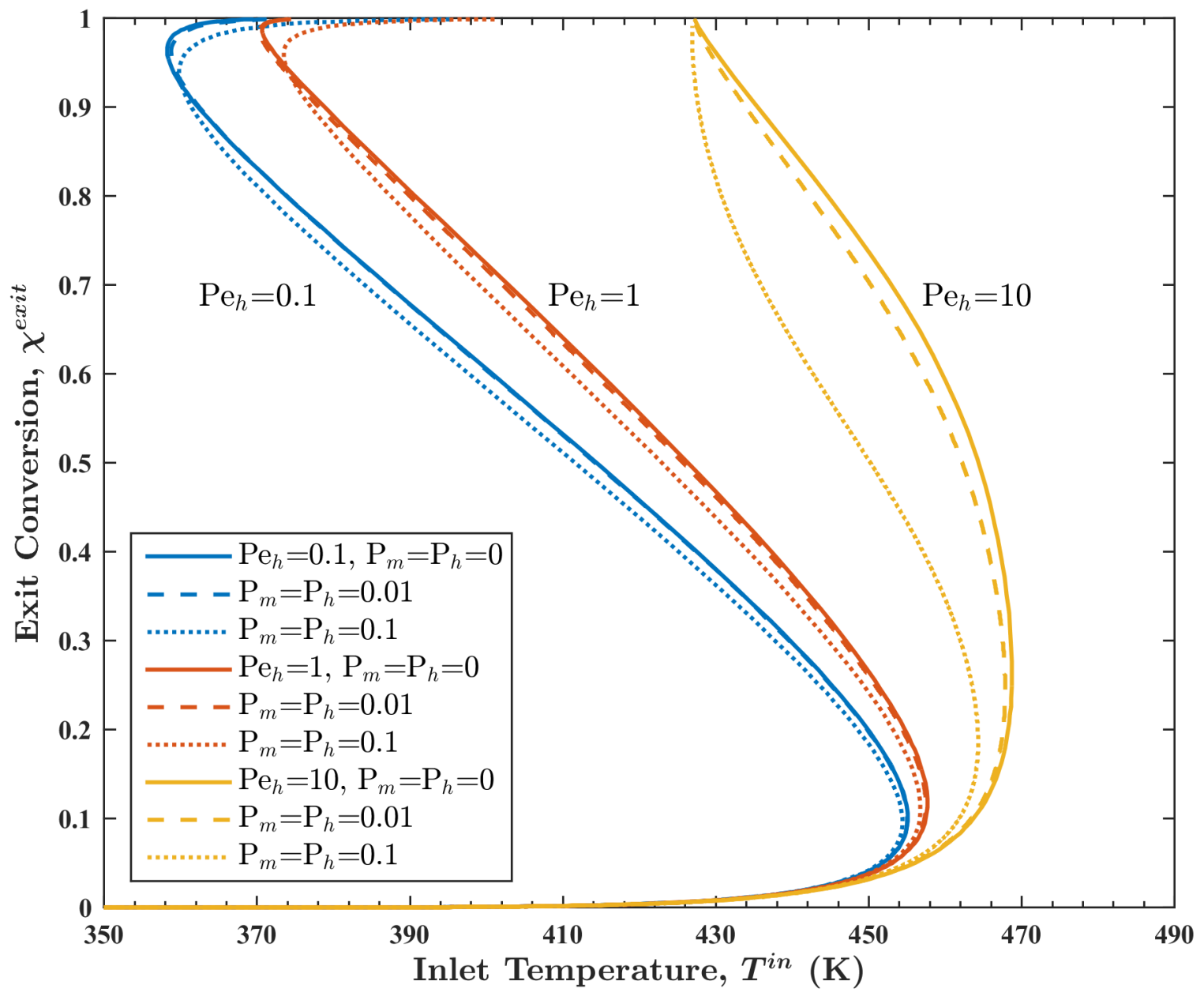

Figure 21: Bifurcation diagrams of steady-state exit conversion versus inlet temperature in adiabatic reactors with first-order kinetics, different axial heat Péclet numbers $\left(P e_{h}\right)$ and different transverse Péclet numbers $\left(P_{m}\right.$ and $\left.P_{h}\right) .\left(E_{a}=100 \mathrm{~kJ} / \mathrm{mol}, D a_{0}=10^{10}, \Delta T_{a d}=200 \mathrm{~K}, P e_{m}=\infty\right)$ 
However, since the transverse Péclet numbers are never actually zero, when the axial Péclet numbers are very large (axial dispersion/conduction effects are negligible) the transverse Péclet numbers (interphase mass and heat transfer) will dominate the reactor behavior. This can be qualitatively understood from the definition of the effective Péclet numbers (Eq. 19). When the axial Péclet number $(P e)$ is very large, the effective Péclet number $\left(P e_{e f f}\right)$ equals to the reciprocal of the transverse Péclet number $(P)$. The usage of the effective Péclet number is only quantitatively correct for small $P$, large $P e$ and small $\Delta T_{a d}$ (such that the model does not exhibit hysteresis), but it can help to predict qualitatively the behavior of a one-dimensional two-phase model (described in Section 2.3.1) even outside its applicable region.

To further illustrate the impact of interphase gradients, we calculated the hysteresis locus for the two-phase model with small $P_{m}$ and $P_{h}$ as shown in Fig. 22. Here, we only solve the $P e_{m}=\infty$

case since it captures the lower boundary of the hysteresis loci with different $P e_{m} / P e_{h}$ ratios (as illustrated in Fig. 13), and thus conservatively defines the boundary of single-solution region. From Fig. 22, we can see that for larger $P e_{h}$ the hysteresis locus for the two-phase model does not increase exponentially as in the pseudo-homogeneous model. Instead, the hysteresis locus will reach a plateau where it is not sensitive to $P e_{h}$, but dominated by $P_{h}$. This trend can also be qualitatively predicted by the effective Péclet number, where the plateau of the hysteresis is determined by the reciprocal of the transverse Péclet number. When $P_{m}$ and $P_{h}$ are small and $P e_{h}$ is not very large, the pseudo-homogeneous model (with lumped effective parameters) gives a very good approximation of the two-phase model.

\section{Impact of heat loss}

So far the analysis is based on either adiabatic reactors (no heat exchange with surroundings) or isothermal reactors (infinitely fast heat exchange with furnace/surroundings). Although a practical reactor is never adiabatic nor isothermal, adiabatic reactors are good approximations for full-scale reactors while lab-scale reactors are closer to isothermal reactors. However, if the adiabatic temperature rise $\Delta T_{a d}$ is too small in the full-scale or too large in the lab-scale reactor, they will diverge from ideal assumptions. Here, we present a preliminary analysis of the impact of heat loss to surroundings 


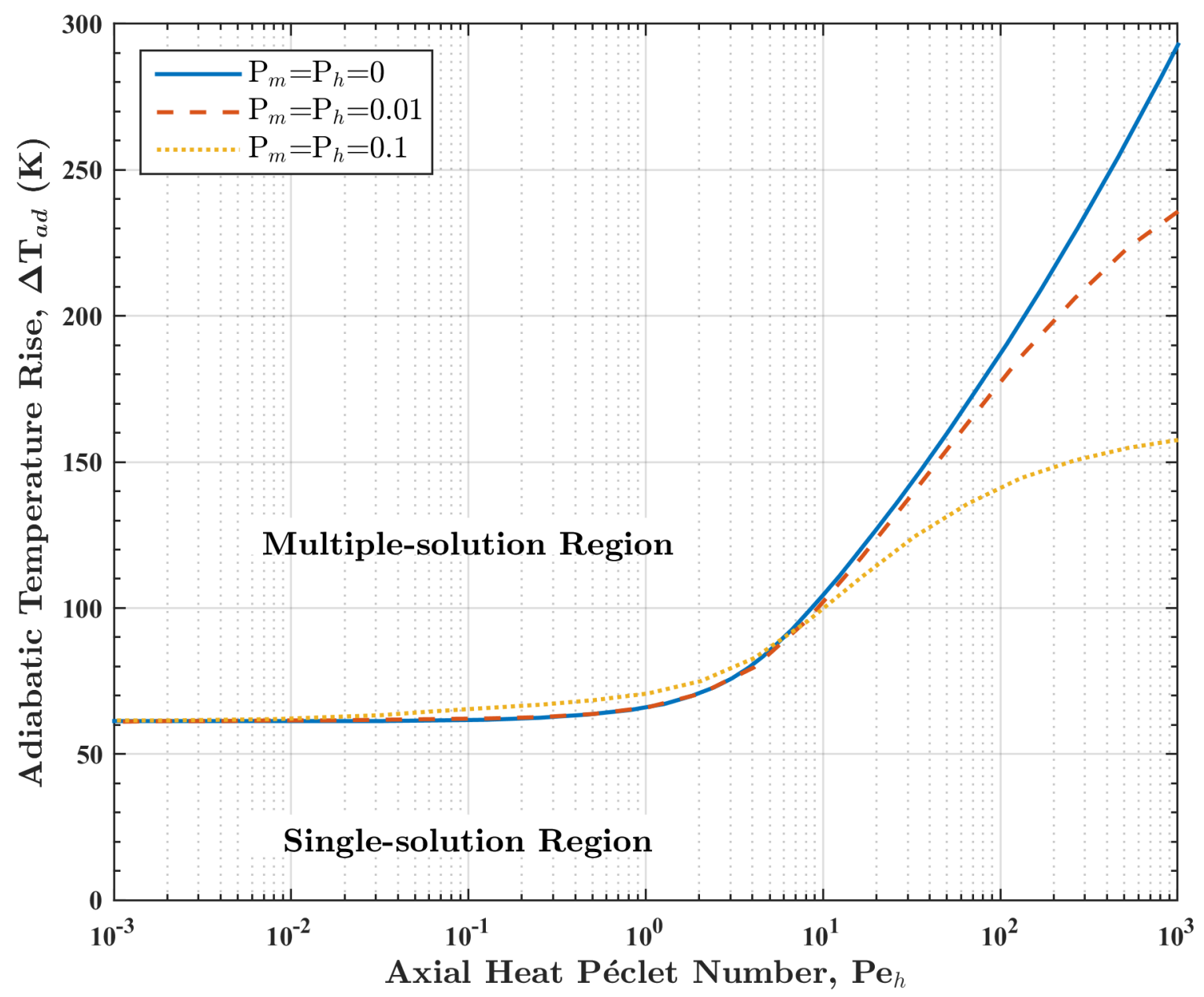

Figure 22: Hysteresis loci of adiabatic reactors with first-order kinetics and different transverse Péclet numbers $\left(P_{m}\right.$ and $\left.P_{h}\right)$ in the $\left(\Delta T_{a d}, P e_{h}\right)$ plane. $\left(E_{a}=100 \mathrm{~kJ} / \mathrm{mol}, D a_{0}=10^{10}, P e_{m}=\infty\right)$ 
on the scale-up performance of monolith reactors.

In general, when there is heat exchange with the surroundings, the temperature in the monolith varies both in the axial and transverse directions and the analysis cannot be based on a single channel. However, when the transverse dimensions of the monolith are small or the effective conductivity in the radial direction is large (or the so called Biot number is small), it is reasonable to assume that the major part of the temperature drop in the radial direction occurs near the wall. We make this assumption here and examine the case of finite external heat transfer by modification of the energy balance of the pseudo-homogeneous model (Eq. 21),

$$
\frac{1}{P e_{h}} \frac{d^{2} \widehat{T}}{d z^{2}}-\frac{d \widehat{T}}{d z}+D a_{0}(1-\chi) \exp \left(\frac{-1 / \beta}{\widehat{T}+\theta^{i n}}\right)-\alpha \Delta \widehat{T}=0
$$

Here, $\Delta \widehat{T}$ is the temperature gradient (or difference) driving the external heat transfer. Further, if we assume that the external/ambient temperature is the same as the inlet temperature, which is consistent with lab experimental operations, we have

$$
\Delta \widehat{T}=\frac{T-T^{i n}}{\Delta T_{a d}}=\widehat{T}
$$

The dimensionless heat loss coefficient $\alpha$ is given by

$$
\alpha=\frac{U a L \delta_{s}}{\rho_{f} C p_{f} \bar{u} R_{\Omega}}
$$

where $U$ is the overall heat transfer coefficient at the outer boundary, $a$ is the external heat transfer area per unit volume of the reactor (For a reactor of circular cross-section, $a=4 / d_{R}$, where $d_{R}$ is the reactor diameter). The dimensionless heat loss coefficient $\alpha$ can also be expressed in terms of characteristic time scales,

$$
\alpha=\frac{U a L \delta_{s}}{\rho_{f} C p_{f} \bar{u} R_{\Omega}}=\frac{\tau}{\tau_{h}} \frac{\delta_{s}}{R_{\Omega}}
$$

where

$$
\tau_{h}=\frac{\rho_{f} C p_{f}}{U a}
$$

is the overall heat transfer time which has a typical range of 0.1 to $1 \mathrm{~s}$ for lab-scale units. The 
parameter $\delta_{s} / R_{\Omega}$ is a fixed geometric factor which is usually in the range $1 / 4$ to $1 / 3$. Since the typical value for space time $\tau$ is $\sim 100 \mathrm{~ms}$, the dimensionless coefficient $\alpha$ ranges from 0.03 to 0.3. Since $\alpha=0.03$ is usually very close to the adiabatic case, $\alpha=0,0.1,1$ and $\infty$ are selected for parametric studies.

The dimensionless heat loss coefficient $\alpha$ can affect the bifurcation behavior of the reactor. When $\alpha=0$, the reactor is operated adiabatically and exhibits ignition/extinction behavior if the adiabatic temperature rise $\Delta T_{a d}$ is large enough. When $\alpha \rightarrow \infty$, the reactor is isothermal and it does not exhibit bifurcation behavior unless there is nonlinearity in the rate expression, e.g. Langmuir-Hinshelwood kinetics. Shown in Fig. 23 are bifurcation diagrams of reactors with $P e_{h}=1$ and different $\alpha$ values. For a fixed $\Delta T_{a d}=200 \mathrm{~K}$, the width of the hysteresis region decreases with $\alpha$ increasing. To better illustrate the effect of external heat transfer, bifurcation sets for different $\alpha$ are calculated. Shown in Fig. 24 are ignition/extinction loci for different $\alpha$ values. The hysteresis region significantly shrinks with increasing $\alpha$. The hysteresis point is also affected by $P e_{h}$ and $\alpha$. Shown in Fig. 25, are hysteresis loci for different $\alpha$ values. With increasing external cooling, larger adiabatic temperature rise is needed for ignition/extinction behavior to appear. Ultimately, when $\alpha \rightarrow \infty$, the hysteresis locus will disappear since there is no nonlinearity in isothermal reactors with linear kinetics. The results shown in Figs. 23-25 indicate that the light-off behavior of laboratory results with different amount of heat losses can be vastly different. This observation is important since most reported laboratory studies do not quantify the heat losses.

As stated earlier, for the case of finite external heat transfer, heat is not well-distributed within the channels and it is best to examine the impact of heat loss by using a two-dimensional model. However, the simpler model analyzed here reveals qualitatively the impact of external heat loss on scale-up. In Section 3 and 4, we have shown that even if lab- and full-scale reactors have the same dimensionless heat loss coefficient $\alpha$ (both zero or both infinity), similarity generally does not exist. In this section, we illustrated that external heat loss can alone invalidate the similarity between the lab-scale and full-scale units when the parameter $\alpha$ does not match in different scales. 


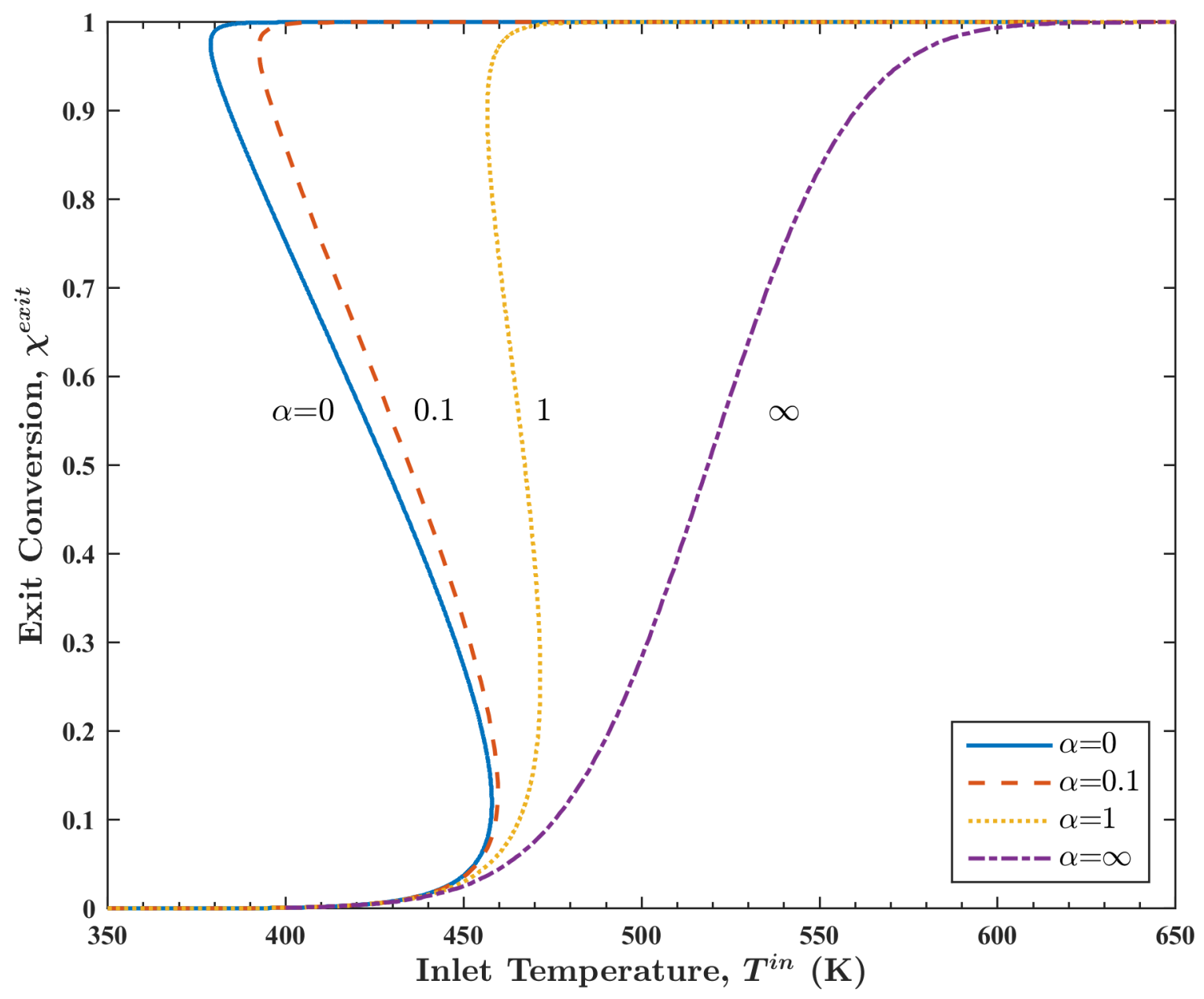

Figure 23: Bifurcation diagrams of steady-state exit conversion versus inlet temperature in reactors with first-order kinetics and different heat loss coefficients $(\alpha)$. $\left(E_{a}=100 \mathrm{~kJ} / \mathrm{mol}, D a_{0}=10^{10}\right.$, $\left.\Delta T_{a d}=200 \mathrm{~K}, P e_{h}=1, P e_{m}=10\right)$ 


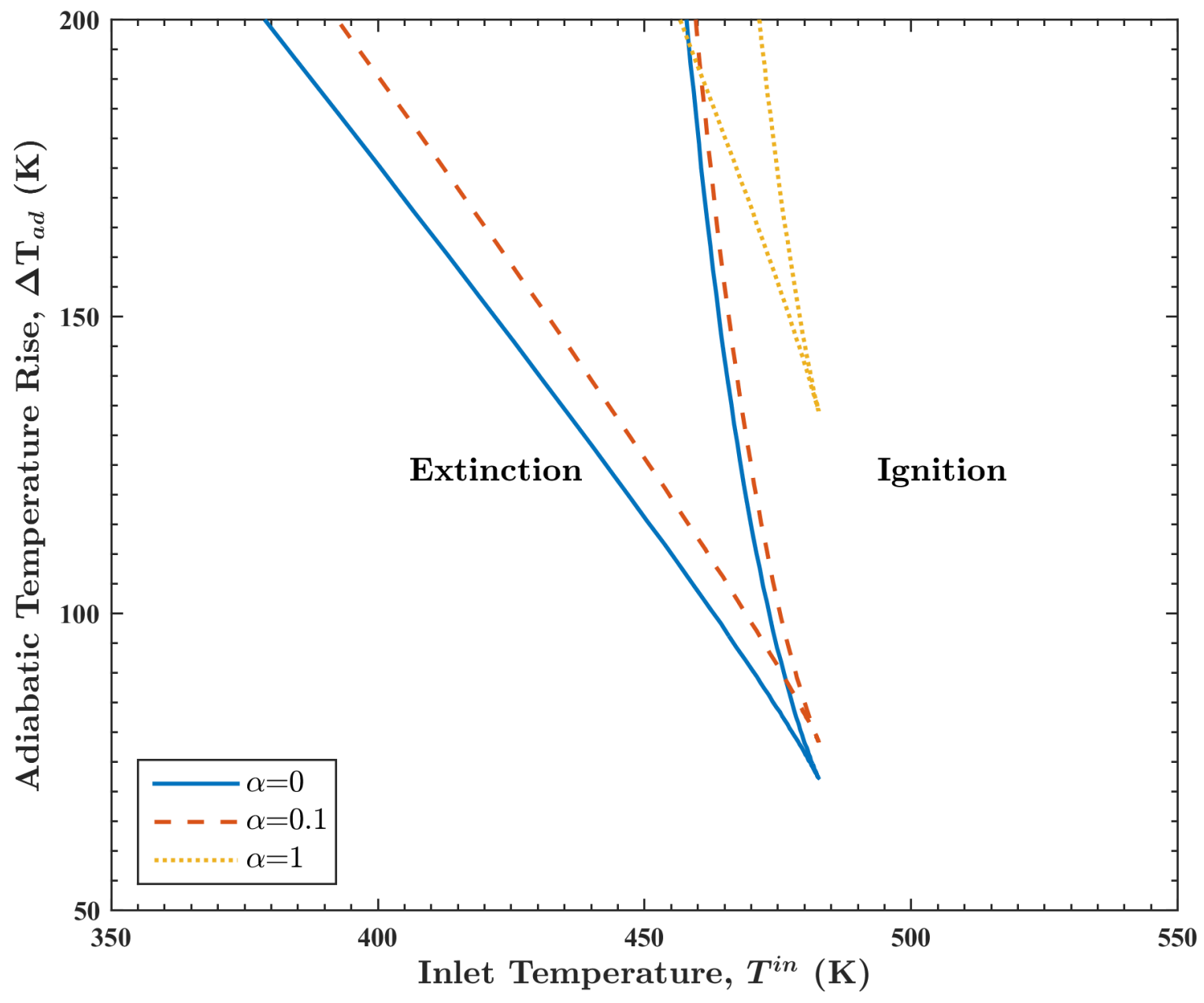

Figure 24: Ignition/extinction loci of reactors with with first-order kinetics and different heat loss coefficients $(\alpha) .\left(E_{a}=100 \mathrm{~kJ} / \mathrm{mol}, D a_{0}=10^{10}, P e_{h}=1, P e_{m}=10\right)$ 


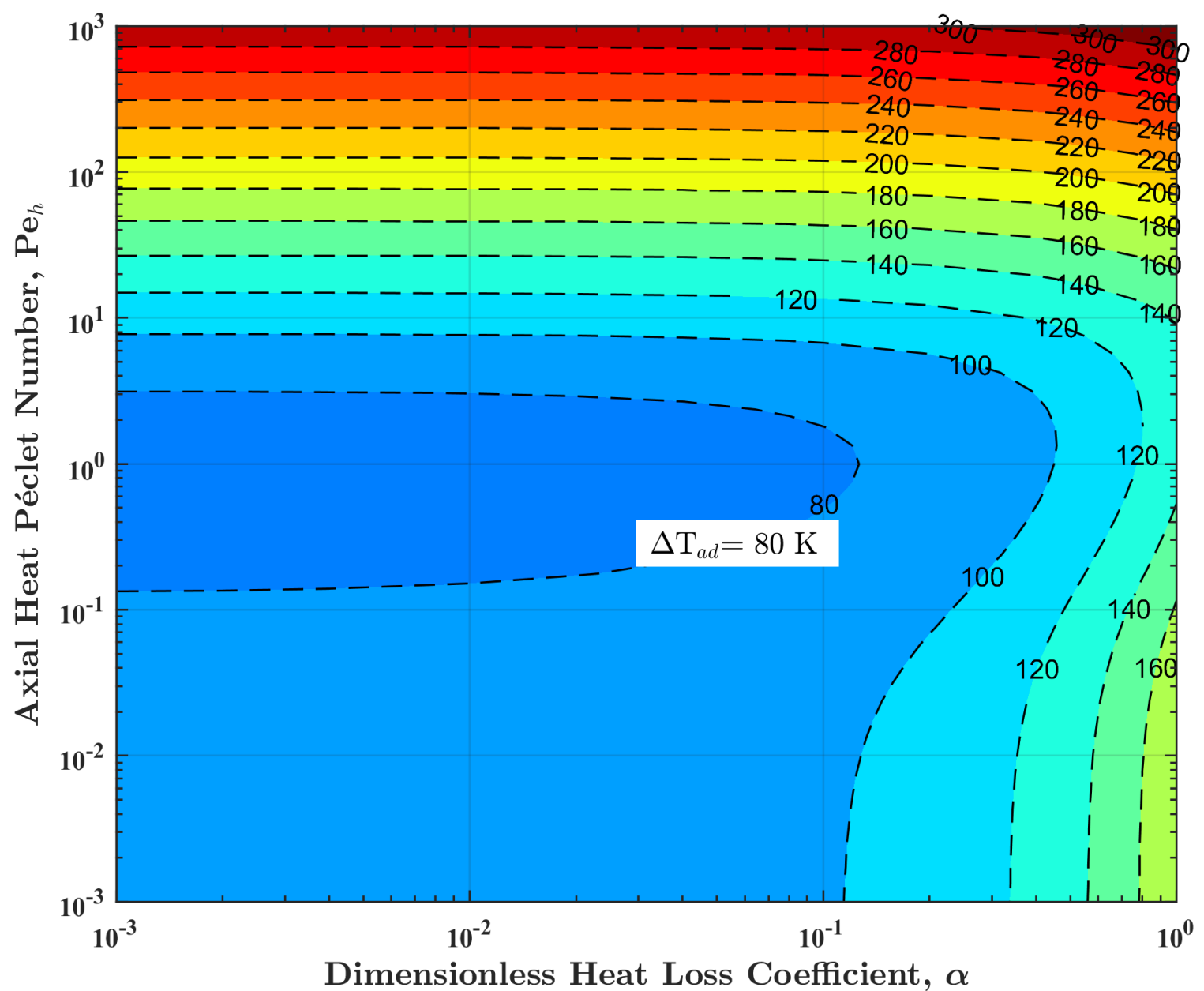

Figure 25: Hysteresis loci of reactors with first-order kinetics, different axial heat Péclet numbers $\left(P e_{h}\right)$ and heat loss coefficients $(\alpha) .\left(E_{a}=100 \mathrm{~kJ} / \mathrm{mol}, D a_{0}=10^{10}, P e_{m} / P e_{h}=10\right)$ 


\section{Conclusions and Discussion}

The main contribution of this work is the detailed investigation of three of the four main reasons for failure of scale-up in monolith reactors used in catalytic after-treatment. The results of our investigation may be summarized as follows: (i) As illustrated in Figs. 3-8, in isothermal reactors the conversion or light-off curves can be different in lab- and full-scale reactors when the effective mass Péclet numbers in the two systems are different with numerical values below the cut-off value of about 10. Equivalently, similarity exists in isothermal reactors with all geometric, kinetic parameters and space times matched only if the impact of mass dispersion is negligible, or the axial mass Péclet numbers in the two systems are much larger than 10 (with this cut-off value increasing further for strongly nonlinear kinetics such as the Langmuir-Hinshelwood with large numerical values for the dimensionless adsorption equilibrium constant). (ii) The results shown in Figs. 10-13 imply that for typical concentrations used in after-treatment systems (with adiabatic temperature rise of about $200 \mathrm{~K})$, the impact of heat dispersion is negligible only if the axial heat Péclet numbers in the two systems exceed the cut-off value of about 100 (and this cut-off value increases exponentially with increase in the adiabatic temperature). (iii) The results in Figs. 23-25 imply that impact of heat loss or heat exchange with surroundings is similar in the lab- and full-scale reactors only if the dimensionless heat loss coefficient $\alpha$ is either close to zero (typically $<0.1$ indicating near adiabatic conditions in both systems) or close to infinity (typically $>100$, indicating near isothermal conditions in both systems). Thus, in the context of catalytic after-treatment reactors, the three parameters $\left(P e_{m}, P e_{h}\right.$ and $\left.\alpha\right)$ determine the conditions for similarity. It should also be pointed out that the current practice is such that the conditions for similarity are not satisfied in most cases (mostly because of the mismatch of these three parameters).

While it is possible to design laboratory reactors such that similarity conditions exist between the two systems, this could make the laboratory experiments somewhat expensive (in terms of consumption of synthetic gases used, amount of catalyst and other resources used). Thus, our view is that it is best to use laboratory experiments to study the kinetics of reactions, evaluate various catalyst formulations, determine reaction mechanisms and so forth. The kinetic models determined from laboratory scale experiments can be combined with estimated (and calibrated) heat and mass 
transfer parameters to simulate the full scale system, and assess the impact of scale-up on reactor performance.

Our analysis in this work dealt mostly with monolith reactors used in after-treatment systems. However, the scale-up principles elucidated here may also be applied for another very common case, namely that of packed-bed catalytic reactors. Here again, similarity may not exist even when one uses the same catalyst composition, particle size and space velocity. The main difference between the monolith and packed-bed reactors is that in the heat and mass transfer correlations used (and hence the formulas for the various effective Péclet numbers). Thus, the main conclusions of this work on scale-up are also applicable to packed-bed as well as other structured catalytic reactors.

\section{Acknowledgements}

We thank Dr. Syed Wahiduzzaman and his colleagues at Gamma Technologies for sharing the Laboratory Reactor Protocol Working Document for calibration of after-treatment reactors. We also thank Drs. R. Blint, Stuart Daw and other CLEERS group members for their comments when the main results of this work were presented in January, 2015. The work of Tian Gu was supported by a grant from National Science Foundation (CMMI-1235461).

\section{Nomenclature}

$\begin{array}{ll}\text { Abbreviations } & \text { Definition } \\ \text { ASC } & \text { Ammonia slip converter } \\ \text { CSTR } & \text { Continuous-flow stirred tank reactor } \\ \text { DOC } & \text { Diesel oxidation converter } \\ \text { LH } & \text { Langmuir-Hinshelwood kinetics } \\ \text { LNT } & \text { Lean-NOx trap } \\ \text { LTR } & \text { Lumped thermal reactor } \\ \text { ODE } & \text { Ordinary differential equation } \\ \text { PFR } & \text { Plug flow reactor }\end{array}$




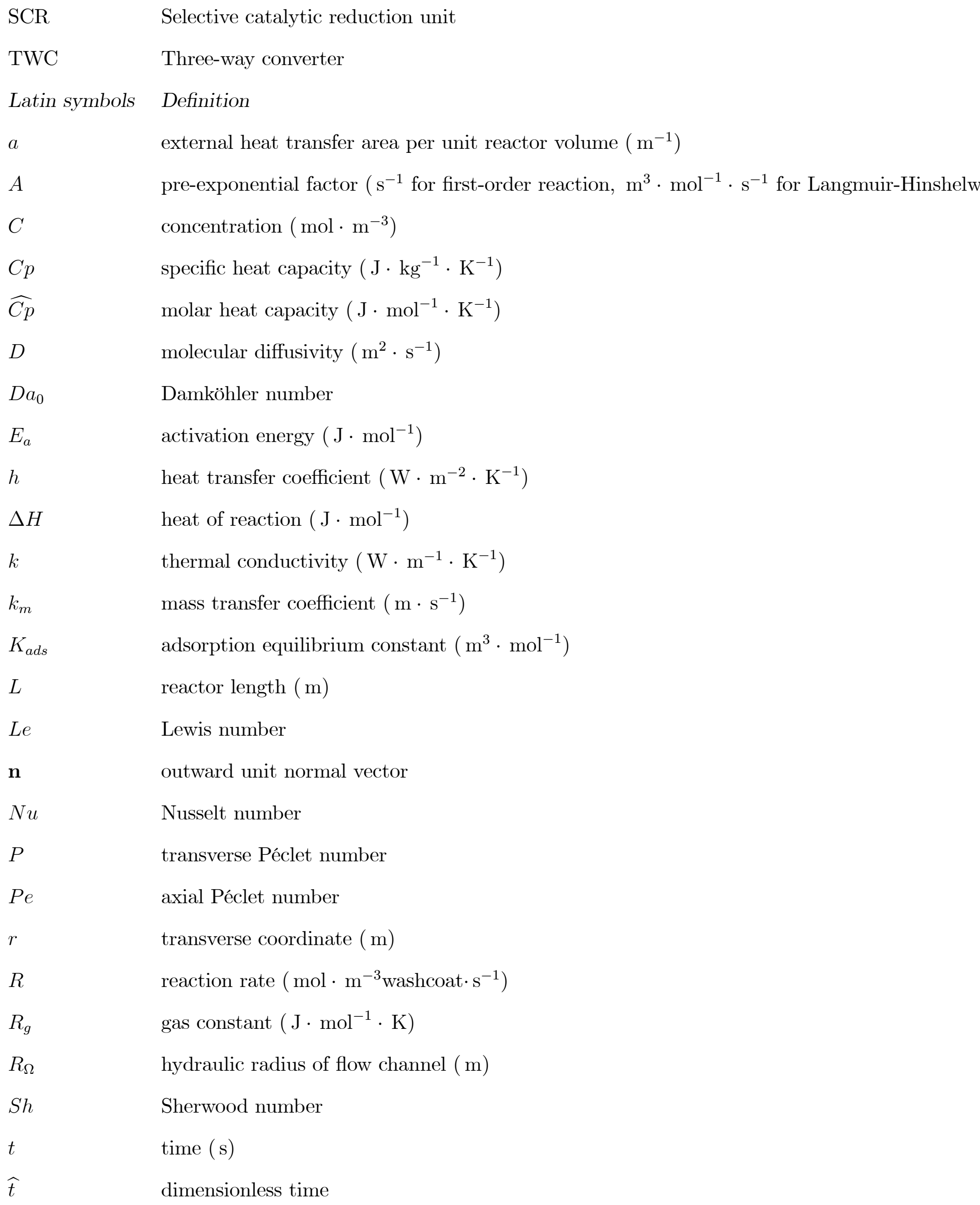




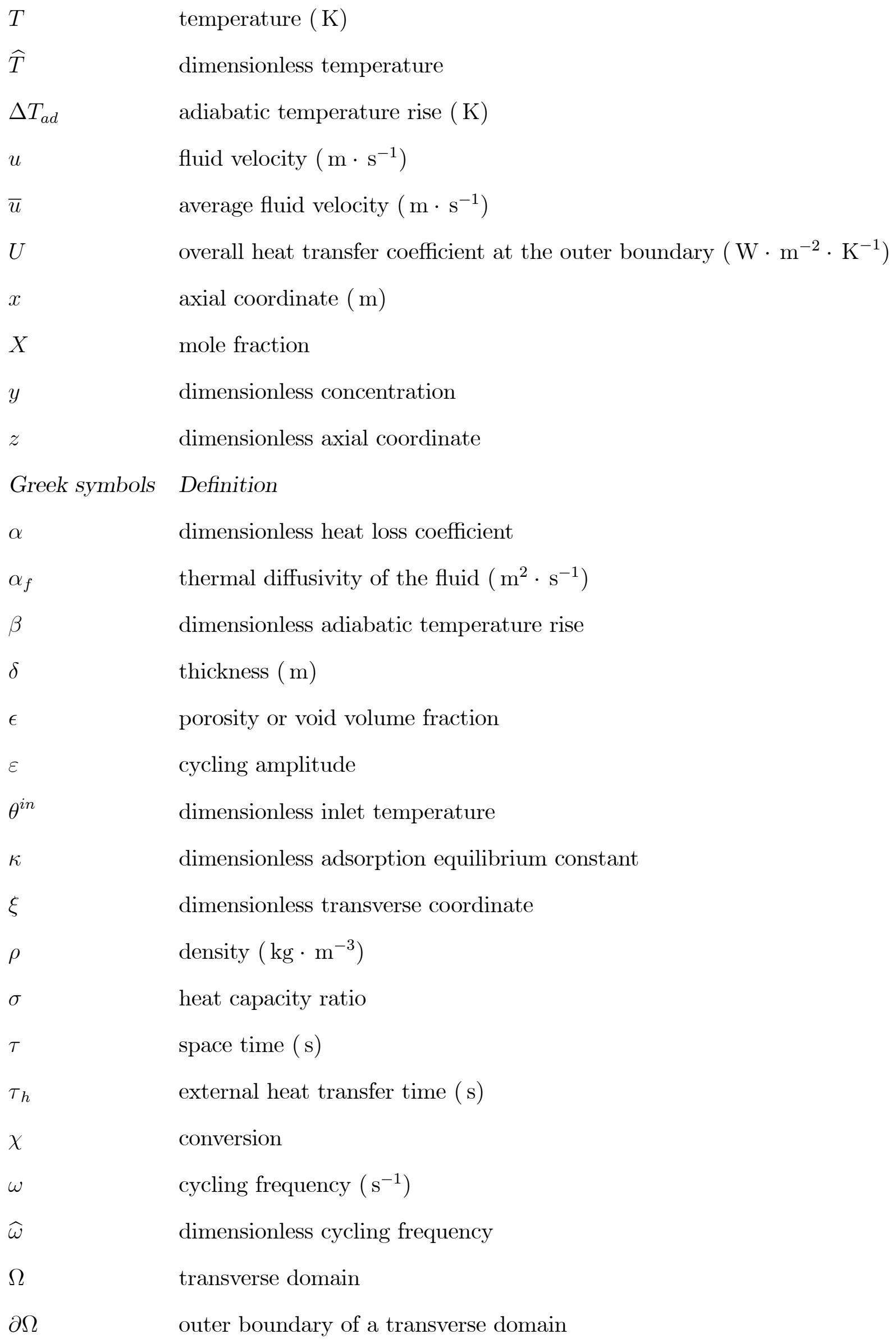




$\begin{array}{ll}\text { Operators } & \text { Definition } \\ \nabla_{\perp} & \text { transverse gradient operator }\left(\mathrm{m}^{-1}\right) \\ \hat{\boldsymbol{\nabla}}_{\perp} & \text { dimensionless transverse gradient operator } \\ \nabla_{\perp}^{2} & \text { transverse Laplacian operator }\left(\mathrm{m}^{-2}\right) \\ \widehat{\nabla}_{\perp}^{2} & \text { dimensionless transverse Laplacian operator } \\ \text { Subscripts } & \text { Definition } \\ e & \text { external mass/heat transfer } \\ f & \text { fluid phase } \\ h & \text { heat transfer } \\ i & \text { internal mass/heat transfer } \\ m & \text { mass transfer } \\ s & \text { solid phase } \\ w & \text { substrate wall } \\ w c & \text { washcoat } \\ \text { Superscripts } & \text { Definition } \\ 0 & \text { initial condition } \\ \text { exit } & \text { exit } \\ \text { in } & \text { inlet condition } \\ \text { LH } & \text { Langmuir-Hinshelwood kinetics } \\ m i d & \text { mid-bed }\end{array}$

\section{References}

[1] http://www.cleers.org.

[2] M. Zlokarnik, Scale-up in Chemical Engineering, Wiley VCH, Weinheim, 2002.

[3] G.F. Froment, K.B. Bischoff, J. De Wilde, Chemical Reactor Analysis and Design, third ed., Wiley, New York, 2010. 
[4] R.M. Heck, R.J. Farrauto, S.T. Gulati, Catalytic Air Pollution Control: Commercial Technology, third ed., Wiley, New York, 2009.

[5] M. Bhattacharya, M.P. Harold, V. Balakotaiah, Shape normalization for catalytic monoliths, Chem. Eng. Sci. 59 (2004) 3737-3766.

[6] V. Balakotaiah, On the relationship between Aris and Sherwood numbers and friction and effectiveness factors, Chem. Eng. Sci. 63 (2008) 5802-5812.

[7] S.Y. Joshi, M.P. Harold, V. Balakotaiah, Overall mass transfer coefficients and controlling regimes in catalytic monoliths, Chem. Eng. Sci. 65 (2010) 1729-1747.

[8] S.R. Gundlapally, V. Balakotaiah, Heat and mass transfer correlations and bifurcation analysis of catalytic monoliths with developing flows, Chem. Eng. Sci. 66 (2011) 1879-1892.

[9] P. Kumar, I. Makki, J. Kerns, K. Grigoriadis, M. Franchek, V. Balakotaiah, A low-dimensional model for describing the oxygen storage capacity and transient behavior of a three-way catalytic converter, Chem. Eng. Sci. 73 (2012) 373-387.

[10] P. Kumar, T. Gu, K. Grigoriadis, M. Franchek, V. Balakotaiah, Spatio-temporal dynamics of oxygen storage and release in a three-way catalytic converter. Chem. Eng. Sci. 111 (2014) $180-190$.

[11] S.R. Gundlapally, V. Balakotaiah, Analysis of the effect of substrate material on the steady-state and transient performance of monolith reactors, Chem. Eng. Sci. 92 (2013) 198-210.

[12] H.S. Fogler, Elements of Chemical Reaction Engineering, fourth ed., Prentice Hall, Upper Saddle River, NJ, 2005.

[13] O. Levenspiel, Chemical Reaction Engineering, third ed., Wiley, New York, 1998.

[14] S.E. Voltz, C.R. Morgan, D. Liederman, S.M. Jacob, Kinetic study of carbon monoxide and propylene oxidation on platinum catalysts, Ind. Eng. Chem. Prod. Res. Dev. 12 (1973) 294-301. 
[15] S. Salomons, R.E. Hayes, M. Votsmeier, A. Drochner, H. Vogel, S. Malmberg, J. Gieshoff, On the use of mechanistic CO oxidation models with a platinum monolith catalyst, Appl. Catal. B: Environ. 70 (2007) 305-313.

[16] L. Olsson, R.J. Blint, E. Fridell, Global kinetic model for lean NOx traps, Ind. Eng. Chem. Res. 44 (2005) 3021-3032.

[17] V.Y. Prikhodko, K. Nguyen, J.S. Choi, C.S. Daw, Axial length effects on Lean NOx Trap performance, Appl. Catal. B: Environ. 92 (2009) 9-16.

[18] J.E. Etheridge, T.C. Watling, Is reactor light-off data sufficiently discriminating between kinetic parameters to be used for developing kinetic models of automotive exhaust aftertreatment catalysts? The effect of hysteresis induced by strong self inhibition, Chem. Eng. J. 264, (2015) 376-388.

[19] M. Yu, D. Luss, V. Balakotaiah, Analysis of flow distribution and heat transfer in a diesel particulate filter, Chem. Eng. J. 226 (2013) 68-78.

[20] S. Subramanian, V. Balakotaiah, Classification of steady-state and dynamic behavior of distributed reactor models, Chem. Eng. Sci. 51 (1996) 401-421.

[21] D. Luss, V. Balakotaiah, Steady-state multiplicity features of chemical reactors, in: L.K. Doraiswamy, R.A. Mashelkar (Eds.), Frontiers in Chemical Reaction Engineering, Vol. 1, Wiley, New Delhi, 1984, pp. 66-84.

[22] V. Balakotaiah, Structural stability of nonlinear convection-reaction models, Chem. Eng. Educ. 30 (1996) 234-239. 

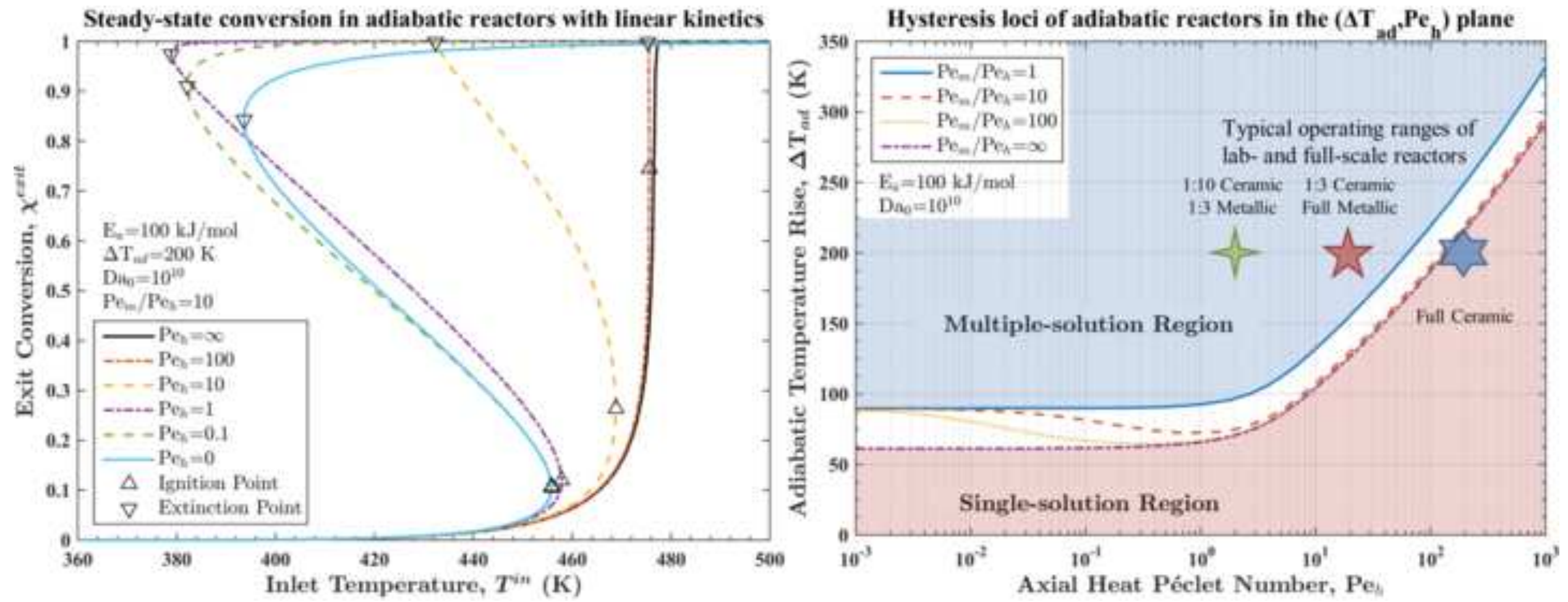